\title{
Partial Breakdown of Input Specificity of STDP at Individual Synapses Promotes New Learning
}

\author{
Maxim Volgushev, ${ }^{1 \star}$ Jen-Yung Chen, ${ }^{2}$ Vladimir Ilin, ${ }^{1}$ Roman Goz, ${ }^{1}$ Marina Chistiakova, ${ }^{1}$ and Maxim Bazhenov ${ }^{2 \star}$ \\ ${ }^{1}$ Department of Psychological Sciences, University of Connecticut, Storrs, Connecticut 06269, and ${ }^{2}$ Department of Medicine, University of California, San \\ Diego, La Jolla, California 92093
}

Hebbian-type learning rules, which underlie learning and refinement of neuronal connectivity, postulate input specificity of synaptic changes. However, theoretical analyses have long appreciated that additional mechanisms, not restricted to activated synapses, are needed to counteract positive feedback imposed by Hebbian-type rules on synaptic weight changes and to achieve stable operation of learning systems. The biological basis of such mechanisms has remained elusive. Here we show that, in layer $2 / 3$ pyramidal neurons from slices of visual cortex of rats, synaptic changes induced at individual synapses by spike timing-dependent plasticity do not strictly follow the input specificity rule. Spike timing-dependent plasticity is accompanied by changes in unpaired synapses: heterosynaptic plasticity. The direction of heterosynaptic changes is weight-dependent, with balanced potentiation and depression, so that the total synaptic input to a cell remains preserved despite potentiation or depression of individual synapses. Importantly, this form of heterosynaptic plasticity is induced at unpaired synapses by the same pattern of postsynaptic activity that induces homosynaptic changes at paired synapses. In computer simulations, we show that experimentally observed heterosynaptic plasticity can indeed serve the theoretically predicted role of robustly preventing runaway dynamics of synaptic weights and activity. Moreover, it endows model neurons and networks with essential computational features: enhancement of synaptic competition, facilitation of the development of specific intrinsic connectivity, and the ability for relearning. We conclude that heterosynaptic plasticity is an inherent property of plastic synapses, crucial for normal operation of learning systems.

Key words: Hebbian learning; heterosynaptic plasticity; homosynaptic plasticity; neocortex; spike timing-dependent plasticity; synaptic plasticity

\section{Significance Statement}

We show that spike timing-dependent plasticity in L2/L3 pyramids from rat visual cortex is accompanied by plastic changes in unpaired synapses. These heterosynaptic changes are weight-dependent and balanced: individual synapses expressed significant LTP or LTD, but the average over all synapses did not change. Thus, the rule of input specificity breaks down at individual synapses but holds for responses averaged over many inputs. In model neurons and networks, this experimentally characterized form of heterosynaptic plasticity prevents runaway dynamics of synaptic weights and activity, enhances synaptic competition, facilitates development of specific intrinsic connectivity, and enables relearning. This new form of heterosynaptic plasticity represents the cellular basis of a theoretically postulated mechanism, which is additional to Hebbian-type rules, and is necessary for stable operation of learning systems.

\section{Introduction}

Plasticity is a universal property of synapses, vital for fundamental operations of the brain. Our present view of plasticity is heavily biased toward input-specific (i.e., homosynaptic) changes, which require presynaptic activation of the synapse during the induction. Indeed, Hebbian-type homosynaptic plasticity represents a cellular mechanism for associative learning and refinement of connectivity. However, theoretical and modeling analyses have long recognized that Hebbian-type rules alone make learning systems unstable and support only a weak degree

The authors declare no competing financial interests.

${ }^{*}$ M.V. and M.B. contributed equally to this study as cosenior authors.

Correspondence should be addressed to Dr. Maxim Bazhenov, Department of Medicine, University of California,

San Diego, La Jolla, CA 92093. E-mail: bazhenov@salk.edu.

V. Ilin's present address: Department of Neurology, University of Pittsburgh, Pittsburgh PA 15260

DOI:10.1523/JNEUROSCI.0552-16.2016

Copyright $\odot 2016$ the authors $\quad 0270-6474 / 16 / 368842-14 \$ 15.00 / 0$ 
of synaptic competition (von der Malsburg, 1973; Oja, 1982; van Rossum et al., 2000; Gütig et al., 2003; Babadi and Abbott, 2010). A theoretically suggested mechanism allowing a robust solution to these problems is heterosynaptic plasticity: changes at synapses that were not active during the induction of homosynaptic plasticity. Heterosynaptic plasticity is typically implemented in models as the normalization of synaptic weights, which affects all synapses of a cell (von der Malsburg, 1973; Oja, 1982; K. D. Miller, 1996; for recent review, see Fiete et al., 2010; Chistiakova et al., 2014). Indeed, strong afferent tetanization may lead to heterosynaptic plasticity at synapses located on the dendritic tree far away from the tetanized inputs (Lynch et al., 1977; Dunwiddie and Lynch, 1978; Abraham and Goddard, 1983; Müller and Swandulla, 1995). However, in experiments with spike timing-dependent plasticity (STDP), only local changes at the synapses surrounding activated inputs have been reported (Schuman and Madison, 1994; Engert and Bonhoeffer, 1997; Royer and Paré, 2003). Experimental evidence and characterization of a form of heterosynaptic plasticity that accompanies STDP and occurs cell-wide, thus having potential to play a role in maintaining homeostasis of synaptic weights over all synapses of a cell, are lacking. A possible candidate is a form of plasticity that can be induced in cortical neurons by the purely postsynaptic protocol of intracellular tetanization: bursts of depolarization-induced spikes without any presynaptic stimulation (Volgushev et al., 1997, 2000; Lee et al., 2012; Chen et al., 2013). In this new study, we asked: (1) whether a similar type of heterosynaptic plasticity accompanies the induction of homosynaptic STDP; (2) what the physiological properties of this form of heterosynaptic plasticity are; and (3) which computational roles it could serve. In experiments with layer $2 / 3$ pyramidal neurons from slices of rat visual cortex, we describe and characterize, for the first time, a novel form of heterosynaptic plasticity that accompanies the induction of STDP but occurs in nonactive synapses. Using computer simulations, we demonstrate that this type of experimentally observed plasticity introduces vital computational features to the model neurons and networks. We suggest that heterosynaptic plasticity accompanying induction of homosynaptic changes is a common feature of plastic synapses in the nervous system. It serves theoretically predicted roles in endowing neuronal systems with operational stability, permitting repetitive learning and enabling $d y-$ namic representations of sensory environments.

\section{Materials and Methods}

Slice preparation. All experimental procedures used in this study were in compliance with the National Institutes of Health regulations and were approved by the Institutional Animal Care and Use Committee of the University of Connecticut. Details of slice preparation and recording were similar to those used in previous studies (Volgushev et al., 2000; Lee et al., 2012; Chen et al., 2013). Wistar rats (15-32 d old) of either sex were anesthetized with isoflurane, decapitated, and the brain was quickly removed and placed into an ice-cold oxygenated ACSF solution, containing the following (in $\mathrm{mM}$ ): $125 \mathrm{NaCl}, 25 \mathrm{NaHCO}_{3}, 25$ glucose, $3 \mathrm{KCl}, 1.25 \mathrm{NaH}_{2} \mathrm{PO}_{4}, 2 \mathrm{CaCl}_{2}, 1 \mathrm{MgCl}_{2}$, bubbled with $95 \% \mathrm{O}_{2} / 5 \% \mathrm{CO}_{2}, \mathrm{pH} 7.4$. Coronal slices (350 $\mu \mathrm{m}$ thickness) containing visual or auditory cortex were prepared from the right hemisphere. Slices were allowed to recover for at least an hour at room temperature. For recording, individual slices were transferred to a recording chamber mounted on an Olympus microscope equipped with infrared differential interference contrast optics. In the recording chamber, slices were submerged in oxygenated ACSF at $30^{\circ} \mathrm{C}-32^{\circ} \mathrm{C}$.

Intracellular recording and synaptic stimulation. Intracellular recordings in the whole-cell configuration were made from layer 2/3 pyramidal cells from visual or auditory cortex using patch electrodes $(4-7 \mathrm{~m} \Omega)$ filled with a potassium-gluconate-based solution, containing the following (in $\mathrm{mm}$ ): $130 \mathrm{~K}$-gluconate, $20 \mathrm{KCl}, 10 \mathrm{HEPES}, 10 \mathrm{Na}-$ phosphocreatine, $4 \mathrm{Mg}$-ATP, $0.3 \mathrm{Na}_{2}$-GTP, pH 7.4 with $\mathrm{KOH}$. Layer 2/3 pyramidal neurons were selected for recording using differential interference contrast microscopy. Our previous work with biocytin labeling and morphological reconstruction of recorded neurons demonstrated reliability of pyramidal cell identification using differential interference contrast microscopy (Volgushev et al., 2000).

Two pairs of stimulating electrodes (S1 and S2) were placed in layer 4, below the layer $2 / 3$ recording site. Stimulation current intensities were adjusted to evoke monosynaptic EPSPs in the layer $2 / 3$ cell. We used paired-pulse stimulation protocol with a $50 \mathrm{~ms}$ interpulse interval. Paired stimuli were applied to S1 and S2 in alternating sequence once per $7.5 \mathrm{~s}$, so that each input was stimulated with paired pulses each $15 \mathrm{~s}$. Small-amplitude hyperpolarizing pulses were applied before S1 stimuli to assess the input resistance. EPSPs in the layer $2 / 3$ cell induced by layer 4 stimulation were recorded during a $10-12 \mathrm{~min}$ control period. Following the control period, a pairing procedure was applied. During the pairing procedure (Chistiakova and Volgushev, 2009), a burst of five action potentials evoked by short depolarizing pulses $(5 \mathrm{~ms}, 100 \mathrm{~Hz})$ was evoked with a $10 \mathrm{~ms}$ delay after stimulation of one of the inputs (either S1 or S2, alternating in different neurons). The current intensity (0.4 nA-1.1 nA) was adjusted to reliably evoke spikes in the burst. Only one of the test inputs was stimulated during the pairing. The other input (S2 or S1) was not stimulated during the pairing procedure, and is referred to as "unpaired." The pairing of presynaptic activation with postsynaptic spiking was repeated 50 times (see Fig. 1A). The pattern of postsynaptic firing during the pairing procedure was similar to postsynaptic firing during the purely postsynaptic protocol of intracellular tetanization used in our prior studies of heterosynaptic plasticity (Volgushev et al., 2000; Chistiakova and Volgushev, 2009; Lee et al., 2012; Chen et al., 2013). After the pairing procedure, alternating synaptic stimulation of the two inputs was resumed, and EPSPs evoked by the test stimuli were recorded for another 30-60 min.

Database and data analysis. Electrophysiological results presented here (see Figs. 1, 2) include a total of $N=170$ inputs to 108 neurons. Comparison data for synaptic changes induced by intracellular tetanization (bursts of postsynaptic action potential evoked by short depolarizing pulses without presynaptic stimulation) include a total of $N=136$ inputs $(N=60$ inputs to 41 neurons from Chen et al., 2013 and $N=76$ inputs to 50 neurons from Lee et al., 2012). All inputs included in the analysis fulfilled the following criteria: (1) stability of EPSP amplitudes during the control period, (2) stability of the membrane potential throughout the recording, and (3) stability of the onset latency and kinetics of the rising slope of the EPSP. EPSP amplitudes were measured as the difference between the mean membrane potential during two time windows. The first time window was placed before the EPSP onset, and the second time window was placed just before the peak of the rising slope of the EPSP. Amplitude of the second EPSP in paired-pulse stimulation paradigm was measured using windows of the same duration but shifted by the length of interpulse interval $(50 \mathrm{~ms})$. The paired-pulse ratio (PPR) was calculated as the ratio of averaged amplitude of the EPSP evoked by the second pulse divided by the averaged amplitude of the EPSP evoked by the first pulse.

For assessing changes of synaptic transmission, amplitude of EPSPs evoked by the first stimulus in a pair was used. The magnitude of plastic changes was calculated as the ratio of average EPSP amplitude after the pairing procedure divided by the average EPSP amplitude during the control period. The criterion for plasticity was a significant $(p<0.05$, Student's $t$ test) change in mean EPSP amplitude between the control and postpairing time periods.

Model of pyramidal neuron. For all simulations, we used an established reduced model of a cortical pyramidal cell (Bazhenov et al., 2002; Chen et al., 2012, 2013; Lemieux et al., 2014). This model was first proposed as a reduction of a multicompartmental pyramidal cell model and consists of two electrically coupled compartments, dendritic and axosomatic (Mainen and Sejnowski, 1996). The current balance equations for the two compartments of the model are as follows:

$$
C_{m}\left(d V_{S} / d t\right)=-g\left(V_{S}-V_{D}\right)-I_{S}^{i n t}
$$




$$
C_{m}\left(d V_{D} / d t\right)=g_{\text {leak }}\left(V_{D}-E_{\text {leak }}\right)-g\left(V_{D}-V_{S}\right)-I_{D}^{\text {int }}-I^{\mathrm{syn}}
$$

where $C_{m}$ is the membrane capacitance, $V_{S}$ and $V_{D}$ is the membrane potential in axosomatic and dendritic compartments, respectively, $g$ is conductance between the two compartments, $I_{S}^{\text {int }}$ is the sum of all active intrinsic currents in axosomatic compartment, $I_{D}^{\text {int }}$ is the sum of all active intrinsic currents in dendritic compartment, and $I^{\text {syn }}$ is the sum of synaptic currents. Because the kinetics of all currents in the axosomatic compartment are much faster than the kinetics of all currents in the dendritic compartment, the membrane potential in axosomatic compartment $V_{\mathrm{S}}$ can be set at equilibrium state. Indeed, using singular perturbations analysis, it can be shown that the state variable $V s$ quickly reaches the manifold of slow motion defined by equation $d V s / d t=$ $F(V s)=0$, thus, Equation 1 can be substituted by the following:

$$
g\left(V_{S}-V_{D}\right)=-I_{S}^{i n t}
$$

This reduced model closely matches the spiking patterns of different classes of cells and has been successfully used in previous modeling network studies (Chen et al., 2012, 2013; Lemieux et al., 2014).

In the axosomatic compartment, the model contained fast $\mathrm{Na}^{+}$ current, $I_{\mathrm{Na}}\left(g_{\mathrm{Na}}=3000 \mathrm{mS} / \mathrm{cm}^{2}\right)$, persistent $\mathrm{Na}^{+}$current, $I_{\mathrm{Na}(\mathrm{p})}$ $\left(g_{\mathrm{Na}(\mathrm{p})}=0.07 \mathrm{mS} / \mathrm{cm}^{2}\right)$, and a fast delayed rectifier $\mathrm{K}^{+}$current, $I_{\mathrm{K}}\left(g_{\mathrm{K}}\right.$ $\left.=200 \mathrm{mS} / \mathrm{cm}^{2}\right)$. In the dendritic compartment, the model contained fast $\mathrm{Na}^{+}$current, $I_{\mathrm{Na}}\left(g_{\mathrm{Na}}=1.5 \mathrm{mS} / \mathrm{cm}^{2}\right)$ persistent $\mathrm{Na}^{+}$current, $I_{\mathrm{Na}(\mathrm{p})}\left(g_{\mathrm{Na}(\mathrm{p})}=0.07 \mathrm{mS} / \mathrm{cm}^{2}\right)$, a slow voltage-dependent, noninactivating $\mathrm{K}^{+}$current, $I_{\mathrm{Km}}\left(g_{\mathrm{Km}}=0.01 \mathrm{mS} / \mathrm{cm}^{2}\right)$, a slow $\mathrm{Ca}^{2+}$. dependent $\mathrm{K}^{+}$current, $I_{\mathrm{KCa}}\left(g_{\mathrm{KCa}}=0.3 \mathrm{mS} / \mathrm{cm}^{2}\right)$, a high-threshold $\mathrm{Ca}^{2+}$ current, $I_{\mathrm{HVA}}\left(g_{\mathrm{HVA}}=0.01 \mathrm{mS} / \mathrm{cm}^{2}\right)$, and a $\mathrm{K}^{+}$leak current, $I_{\mathrm{KL}}\left(g_{\mathrm{KL}}=0.0025 \mathrm{mS} / \mathrm{cm}^{2}\right)$. Membrane capacitance was $C_{\mathrm{m}}=0.75$ $\mu \mathrm{F} / \mathrm{cm}^{2}$, and leak conductance was $g_{\mathrm{L}}=0.033 \mathrm{mS} / \mathrm{cm}^{2}$. Equlibrium potentials were as follows: $E_{N a}=50 \mathrm{mV}, E_{\mathrm{K}}=-95 \mathrm{mV}, E_{\text {leak }}=-68$ $\mathrm{mV}, E_{C a}=140 \mathrm{mV}$. For all currents, the voltage and $\mathrm{Ca}^{2+}$-dependent kinetics are listed by Chen et al. (2012).

Synaptic currents. A total of 100 AMPA-type synapses were located at the dendritic compartment (see Fig. 3A). The synaptic current at each synapse was simulated by first-order activation kinetics as follows:

$$
I_{\text {syn }}=W_{\text {syn }}[\mathrm{O}]\left(V-E^{\text {syn }}\right)
$$

where $W_{\text {syn }}$ is synaptic weight and $E^{\text {syn }}$ is the reversal potential $\left(E^{\text {syn }}=\right.$ $0 \mathrm{mV})$. The synaptic weight was defined in the range between $0 \mathrm{mS} / \mathrm{cm}^{2}$ and a maximum of $0.0303 \mathrm{mS} / \mathrm{cm}^{2}$. The initial weights were randomly assigned to the 100 synapses from a Gaussian distribution with mean equaling $0.015 \mathrm{mS} / \mathrm{cm}^{2}$ and SD equaling $0.003 \mathrm{mS} / \mathrm{cm}^{2}$. The fraction of open channels $[\mathrm{O}]$ is calculated according to the following:

$$
\begin{gathered}
d[\mathrm{O}] / d t=\alpha(1-[\mathrm{O}])[\mathrm{T}]-\beta[\mathrm{O}] \\
{[\mathrm{T}]=A H\left(t_{0}+t_{\max }-t\right) H\left(t-t_{0}\right)}
\end{gathered}
$$

where $H$ is Heaviside (step-) function, $t_{0}$ is the time instant of receptor activation, $t$ is simulation time, $A=0.5$, and $t_{\max }=0.3 \mathrm{~ms}$. The rate constants, $\alpha$ and $\beta$, are $1.1 \mathrm{~ms}^{-1}$ and $0.19 \mathrm{~ms}^{-1}$, respectively.

A simple phenomenological model characterizing short-term depression of intracortical excitatory connections was also included in the current model. According to this model, a maximal synaptic conductance was multiplied by the depression variable, $D$, which represents the amount of available synaptic resources. Here, $D=1-\left(1-D_{\mathrm{i}}(1-U)\right)$ $\exp \left(-\left(t-t_{\mathrm{i}}\right) / \tau\right)$, where $U=0.07$ is the fraction of resources used per action potential, $\tau=700 \mathrm{~ms}$ is the time constant of recovery of the synaptic resources, $D_{\mathrm{i}}$ is the value of $D$ immediately before the $i_{\text {th }}$ event, and $\left(t-t_{\mathrm{i}}\right)$ is the time after $i_{\text {th }}$ event.

STDP. STDP was implemented as in previous modeling studies (Song et al., 2000; Kempter et al., 2001; Rubin et al., 2001; Song and Abbott, 2001). It is assumed that every synaptic pairing event can potentially trigger a change of synaptic weight and that the direction and amount of synaptic modification are determined by the time difference between presynaptic and postsynaptic spiking events. If a presynaptic spike occurred before a postsynaptic spike within a certain time window, the synaptic weight increased. If a presynaptic spike occurred after a post- synaptic spike within a certain time window, the synaptic weight decreased. This spike timing-dependent plasticity was implemented by the following equations:

$$
\begin{aligned}
& \mathrm{dW}_{\text {syn }^{+}}=a^{+}\left(\exp \left(-\left(t^{\text {post }}-t^{\text {pre }}\right) / \tau^{+}\right)\right) \\
& \mathrm{dW}_{\text {syn }}=-a^{-}\left(\exp \left(\left(t^{\text {post }}-t^{\text {pre }}\right) / \tau^{-}\right)\right)
\end{aligned}
$$

In Equations 7 and $8, \mathrm{dW}_{\text {syn }}{ }^{+}$and $\mathrm{dW}_{\text {syn }}{ }^{-}$are the change of synaptic weight, $a^{+}$and $a^{-}$are the maximal amplitude of potentiation and depression that could be induced by a single pair of presynaptic and postsynaptic spikes, $t^{\text {post }}$ and $t^{\text {pre }}$ are the timing of occurrence of postsynaptic and presynaptic spikes, and $\tau^{+}$and $\tau^{-}$are the time constants of synaptic potentiation and synaptic depression. As a standard setting, we used STDP with symmetrical potentiation and depression windows: $a^{+}$and $a^{-}$ $=0.001 \mathrm{mS} / \mathrm{cm}^{2}$, and $\tau^{+}$and $\tau^{-}=20 \mathrm{~ms}$ (Fig. 3B1).

Heterosynaptic plasticity. Heterosynaptic plasticity was implemented according to rules derived from the results of in vitro experiments presented in this paper (see Figs. 1,2) and results of our prior studies (Volgushev et al., 2000; Chistiakova and Volgushev, 2009; Lee et al., 2012). In slice experiments, induction of heterosynaptic plasticity required a rise of intracellular $\left[\mathrm{Ca}^{2+}\right]$ in the postsynaptic neuron (Lee et al., 2012). This was implemented in the model by introducing a calcium threshold $(0.4 \mu \mathrm{M}$ in the standard model, tested range between $0.2 \mu \mathrm{M}$ and $0.8 \mu \mathrm{M}$ ). Our experimental results show that the effect of heterosynaptic plasticity depended on initial state of synapse: synapses with initially low release probability have a tendency to undergo potentiation, whereas synapses with initially high release probability tended to be depressed or did not change after intracellular tetanization (Volgushev et al., 2000; Chistiakova and Volgushev, 2009; Lee et al., 2012). Dependence of heterosynaptic plasticity on intracellular $\left[\mathrm{Ca}^{2+}\right]$ and initial synaptic weight was implemented as follows. When intracellular $\left[\mathrm{Ca}^{2+}\right]$ exceeded the threshold level, the probability of occurrence of heterosynaptic plasticity, Prob, was calculated as follows:

$$
\operatorname{Prob}\left(W_{\text {syn }}\right)=3000 \times\left(W_{\text {syn }}-0.015\right)^{2}+0.1
$$

where $W_{\text {syn }}$ represents current value of synaptic weight which ranged from 0 to $0.03 \mathrm{mS} / \mathrm{cm}^{2}$. According to Equation 9, synapses with intermediate weights had lower probability to change, whereas synapses with high or low weights had higher probability to change. Next, the magnitude of synaptic change $d W_{\text {syn }}$ was calculated as follows:

$$
\begin{aligned}
d W_{\text {syn }}=\left(\frac{1}{1+\exp \left(\left(W_{\text {syn }}-\left(0.5 \times W_{\text {max }}\right)\right) \times 100\right)}\right. & \\
& -0.5+\sigma \times 0.02) \times 0.0001
\end{aligned}
$$

where $W_{\text {syn }}$ is the current synaptic weight, $W_{\max }$ is the maximum value of synaptic weight, and $\sigma$ is a random variable derived from the standard normal distribution with 0 mean and SD of 3 . The weight dependence of the probability and magnitude of heterosynaptic changes are shown in Figure 3B2.

Neural network model. Two neural networks consisting of 10 pyramidal neurons (see Fig. 7) or 100 pyramidal neurons (see Fig. 8) were constructed. In both networks, connections between neurons were excitatory (see Eq. 4) and random with connecting probability $p=0.5$. Each neuron thus contained two sets of synapses: those that received external spike trains and those that received intrinsic spike events from other neurons in the network. In both sets of synapses, STDP rules were implemented with or without heterosynaptic plasticity (depending on the experimental conditions).

Input spike trains. All external synapses in a single-cell or network model were stimulated by independent spike trains representing presynaptic activity. In different experiments, these spike trains had different mean frequency and/or different level of correlation among them. Spike trains were generated as follows. First, multiple spike train templates with independent Poisson distributed interspike intervals were generated. Next, an individual spike train was created by randomly selecting spiking 

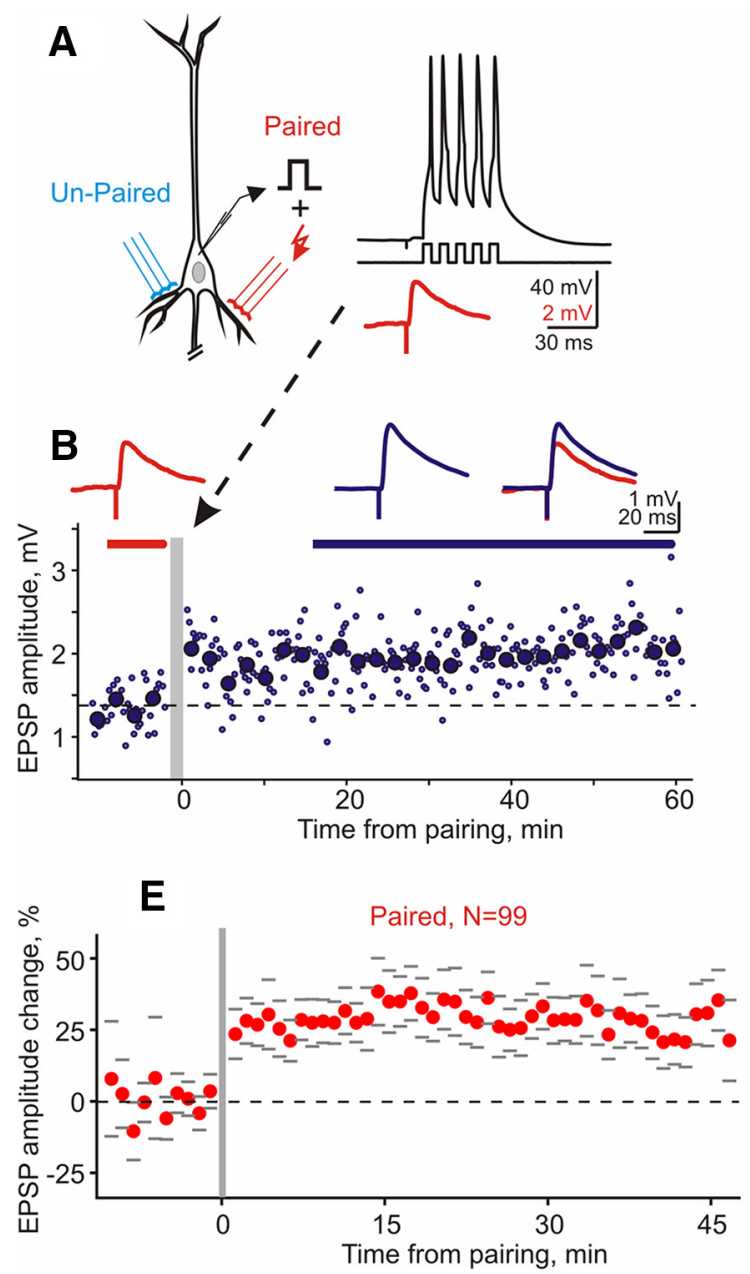
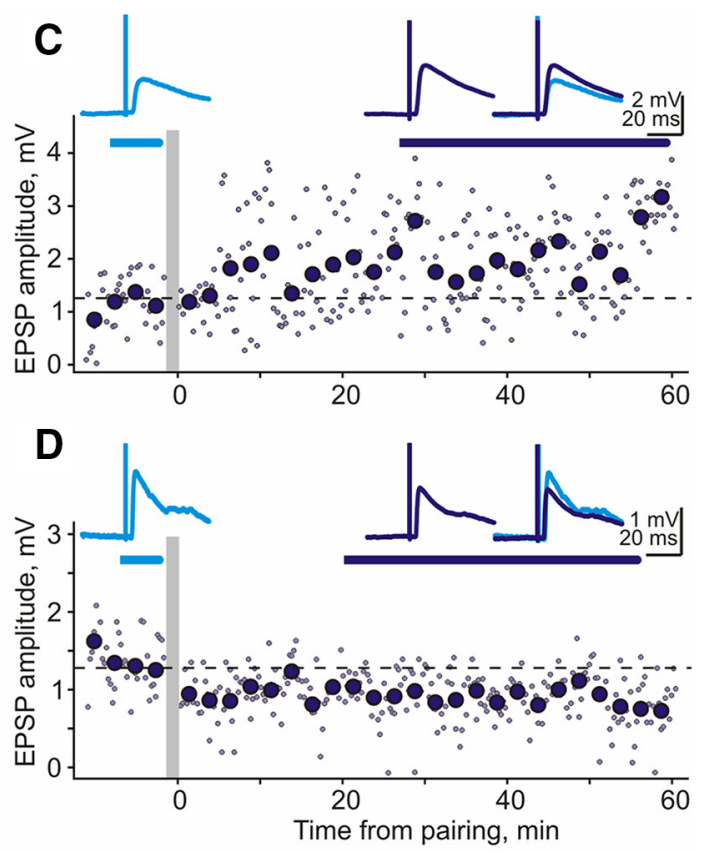

$\mathbf{F}$

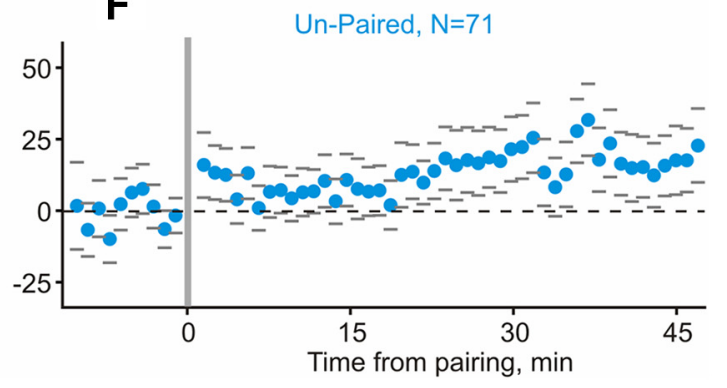

Figure 1. Plasticity induced by a pairing procedure (STDP) in paired (homosynaptic) and unpaired (heterosynaptic) synapses. $A$, A scheme of the STDP protocol. A burst of five action potentials induced by short depolarizing pulses $(5 \mathrm{~ms}, 100 \mathrm{~Hz}$ ) was applied with a $10 \mathrm{~ms}$ delay after stimulation of one synaptic input ("Paired"). The pairing was repeated 50 times. The other synaptic input ("Unpaired") was not stimulated during the pairing, so plastic changes in these synapses were heterosynaptic. $\boldsymbol{B}-\boldsymbol{D}$, Examples of homosynaptic LTP in paired synapses (B) as well as heterosynaptic LTP $(\boldsymbol{C})$ and heterosynaptic LTD $(\boldsymbol{D})$ in unpaired synapses induced by the STDP protocol in layer $2 / 3$ pyramidal neurons. Red or cyan traces represent EPSPs before the pairing. Dark blue traces represent EPSPs after the pairing (averaged over the periods marked by horizontal bars). Time course shows amplitudes of individual EPSPs (dots) and averages of 10 consecutive responses (filled circles). $\boldsymbol{E}, \boldsymbol{F}$, Time course of amplitude changes averaged over 99 paired synaptic inputs ( $\boldsymbol{E}$; homosynaptic changes) and 71 unpaired inputs ( $\boldsymbol{F}$; heterosynaptic changes). Filled circles represent 1 min averages. Horizontal bars indicate \pm SEM. $\boldsymbol{B}-\boldsymbol{F}$, Gray vertical bars represent the period of the STDP procedure. Dashed horizontal lines indicate averaged EPSP amplitude in control.

events from these templates. The frequency of firing of input spike trains was controlled by the total number of spikes selected from these templates in a certain time interval. The degree of correlation among input spike trains was controlled by the total number of templates used. When the number of templates was increased, the correlation among input spike trains was reduced. After input spike trains were generated, the cross-correlation among them was calculated.

In simulations for Figures 6-8, inputs to a model neuron were divided in groups representing "stimuli." Each stimulus was uniquely represented by higher average frequency $(4 \mathrm{~Hz})$ and correlation $(0.91)$ of input spike trains for one group of synapses compared with the background activity at the other inputs $(1 \mathrm{~Hz}$, averaged correlation 0.34$)$.

Principal component analysis. To quantify the network performance in the discrimination task we used principal component analysis. We recorded spike responses of the model network to 10 individual realizations of each stimulus S1-S5 before, during and after the training. Synaptic plasticity was turned off during the test. We counted spikes generated by each neuron during $25 \mathrm{~s}$ of response and used principal component analysis to project 10-dimensional space of the network responses to the first three principal components. In Figure 7E-I, each dot represents response of the entire network to one stimulus presentation.

\section{Results}

Heterosynaptic changes accompany pairing-induced homosynaptic plasticity

Our previous in vitro studies revealed that bursts of spikes evoked in a neuron by depolarizing pulses without presynaptic stimulation (intracellular tetanization) led to heterosynaptic plasticity: weight-dependent changes at unstimulated synapses (Chistiakova and Volgushev, 2009; Chen et al., 2013; Chistiakova et al., 2014). However, the vast majority of experimental paradigms for studying synaptic plasticity include presynaptic activation of at least some synaptic inputs to a cell. To establish a link between heterosynaptic plasticity induced by purely postsynaptic intracellular tetanization and plasticity induced by conventional protocols, we asked whether heterosynaptic changes at nonactivated synapses can be induced in parallel with the induction of associative (Hebbian-type) plasticity. To test this hypothesis, we recorded EPSPs evoked by stimulation of two independent synaptic inputs in layer $2 / 3$ pyramidal neurons in slices of rat visual cortex. We used a well-established STDP protocol to induce LTP at one 
A

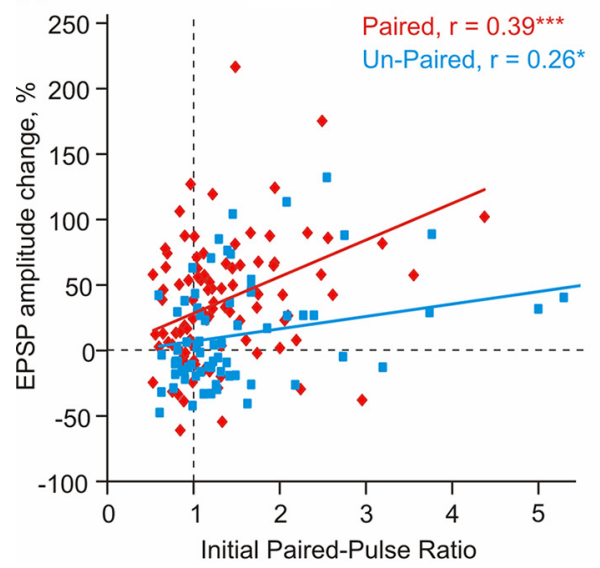

B

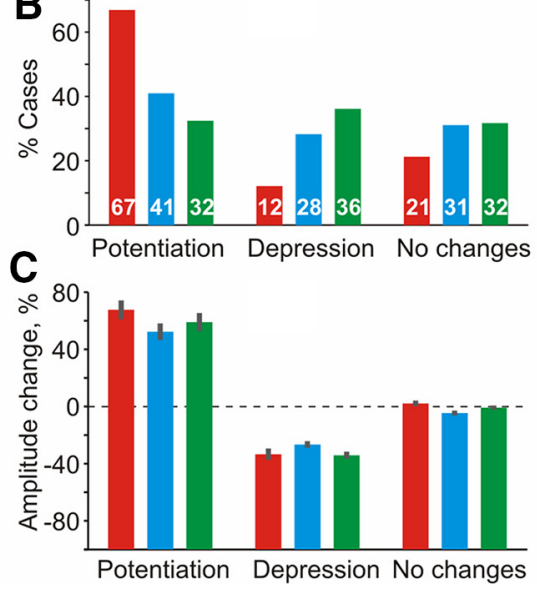

Paired, $\mathrm{N}=99$

Un-Paired, $\mathrm{N}=71$

Intracellular

Tetanization, $\mathrm{N}=136$

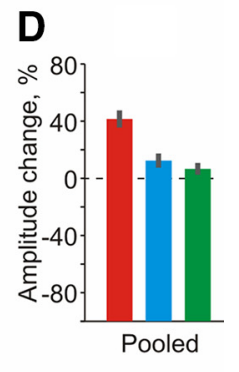

Figure 2. Homosynaptic and heterosynaptic plasticity induced by the STDP procedure: Population analysis. $A$, Correlation between long-term changes of EPSP amplitude and initial PPR for paired $(N=99$, red, diamonds, $r=0.39, p<0.001)$, and unpaired ( $N=71$, cyan, squares, $r=0.26, p<0.05)$ synaptic inputs. $B$, Percentage of cases of LTP, LTD, or no changes in paired ( $N=99$, red) and in unpaired ( $N=71$, cyan) synaptic inputs. For comparison, data for plasticity induced by intracellular tetanization are shown ( $N=136$, green; data from Chen et al., 2013). Columns and numbers within them show percentage of inputs in each of the three experimental groups (paired, unpaired, and intracellular tetanization, color-coded; $\chi^{2}$ test, $p<0.001$ for paired vs unpaired, and paired vs intracellular tetanization). C, EPSP amplitude changes, in percentage of control, in synapses with significant LTP, LTD, or no significant changes. Data are mean \pm SEM. Data for the three experimental groups (paired, unpaired, and intracellular tetanization) are color-coded. D, Averaged EPSP amplitude changes in three experimental groups (paired, unpaired, and intracellular tetanization).

of the inputs to the neuron (Fig. 1A). The STDP protocol induced a robust LTP in the paired synaptic inputs (149\% of control in Fig. $1 B$, example; and $141.9 \pm 57.9 \%$, average $\pm \mathrm{SD}$, in $N=99$ experiments, $p<0.001$, Figs. $1 E, 2 D)$. Importantly, significant changes of response amplitude $(p<0.05)$ were not restricted to the paired synapses but could occur also at unpaired synapses, which were not stimulated during the pairing procedure (Fig. $1 A$, cyan). Individual unpaired synaptic inputs showed significant LTP (Fig. $1 C$; $153 \%$ of control) or LTD (Fig. 1D; 68\% of control), or did not change. However, consistent with the notion of input specificity of synaptic plasticity (Bliss and Collingridge, 1993), changes of EPSP amplitudes averaged over all unpaired synaptic inputs were not significant (112.7 $\pm 40.1 \%$ of control, $N=71$, not significant; Figs. $1 F, 2 D)$. We interpret these results as indicative of the balanced nature of synaptic changes at unpaired synapses, so that potentiation and depression cancelled each other on the average. This interpretation is consistent with a metaanalysis of published data (Chistiakova et al., 2014).

Next, we asked what the physiological properties of this form of heterosynaptic plasticity are. Plastic changes in both paired and unpaired synapses correlated with the initial PPR (the ratio of the amplitude of responses evoked by the second and the first pulses in a paired-pulse paradigm). PPR is an index of synaptic release inversely related to the release probability. We found that synapses with initially high PPR (low release probability, weak synapses) had a tendency to be potentiated, whereas synapses with initially low PPR (high release probability, strong synapses) had a tendency to be depressed or unchanged (Fig. $2 A ; r=0.39$, $N=99, p<0.001$ for paired inputs; and $r=0.26, N=71, p<$ 0.05 for unpaired inputs). This weight dependence of the direction and magnitude of synaptic changes was similar to weight dependence of plastic changes induced by postsynaptic intracellular tetanization described in our previous studies (Volgushev et al., 2000; Chistiakova and Volgushev, 2009; Lee et al., 2012; Chen et al., 2013; Chistiakova et al., 2014). The pairing procedure shifted the entire distribution of synaptic changes upward toward potentiation, as indicated by the upward shift of the regression line for the paired synapses (Fig. 2A). This interpretation was substantiated by the increased frequency of occurrence of LTP and decreased frequency of LTD in the paired synapses $(67 \%, 66$ of 99 cases of LTP; and 12\%, 12 of 99 of LTD) compared with unpaired synapses (41\%, 29 of 71 cases of LTP; and 28\%, 20 of 71 of LTD, $p<0.001$ for paired vs unpaired comparison; Fig. $2 B$ ).

Frequency of occurrence of LTP and LTD in the unpaired synapses in STDP experiments was comparable with that observed after purely postsynaptic intracellular tetanization (Fig. $2 B$, cyan and green bars; $\chi^{2}$ test, not significant for comparison of unpaired vs intracellular tetanization). Interestingly, the magnitude of potentiation or depression in synapses, which showed significant amplitude changes $(p<0.05)$, was similar in all three groups: paired, unpaired, and after intracellular tetanization (Fig. 2C). Thus, significant averaged LTP of paired inputs was due to the more frequent occurrence of LTP and less frequent LTD induced by the pairing procedure (Fig. $2 B ; p<0.001$ for paired vs unpaired, and paired vs intracellular tetanization) and not due to a greater magnitude of plastic changes in individual synapses (Fig. $2 C$ ). During a pairing procedure, only a small portion of inputs at a given cell are stimulated. Therefore, overall synaptic changes should be dominated by plasticity in unpaired synapses. Because plastic changes in unpaired synapses are balanced on average, the cumulative synaptic input to the cell will change little, close to the average of changes seen in the unpaired synapses.

\section{Homosynaptic (STDP) and heterosynaptic plasticity in a neuron model}

To study how synaptic weights are determined by the interplay between homosynaptic and heterosynaptic plasticity, we built a biophysically realistic neuron model of pyramidal cell receiving 100 excitatory synaptic inputs (Fig. 3A). Homosynaptic changes followed STDP rules (Fig. 3B1). Heterosynaptic plasticity was implemented in accordance with experimental data described above, with weight-dependent probability of changes (Fig. 3B2, blue line, Eq. 9) and weight-dependent direction and amplitude of changes (Fig. 3B2, green line, Eq. 10). Figure 3 illustrates changes of synaptic weights induced by a "pairing" protocol, in which activation of a portion of synapses (synapses 91-100) was 
A

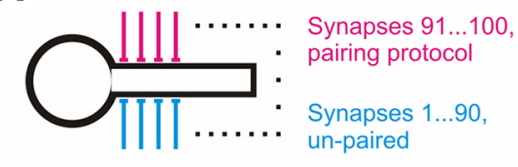

B1

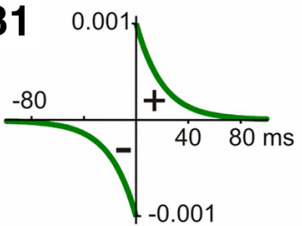

B2

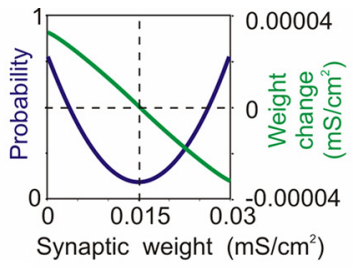

C

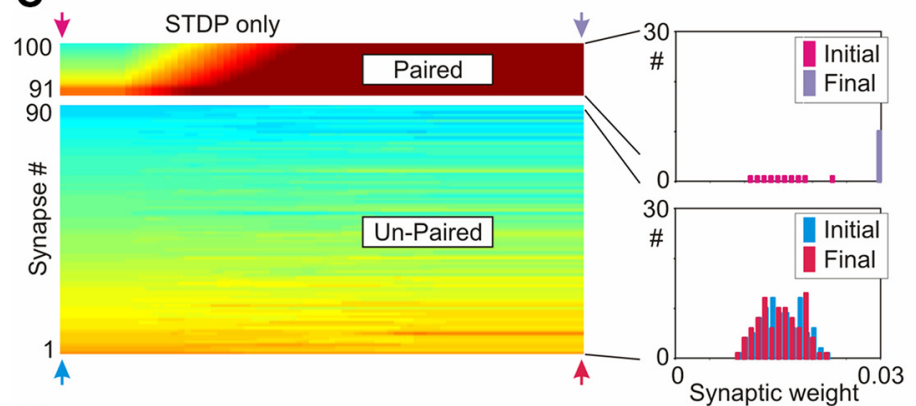

E $\left(\mathrm{mS} / \mathrm{cm}^{2}\right)$

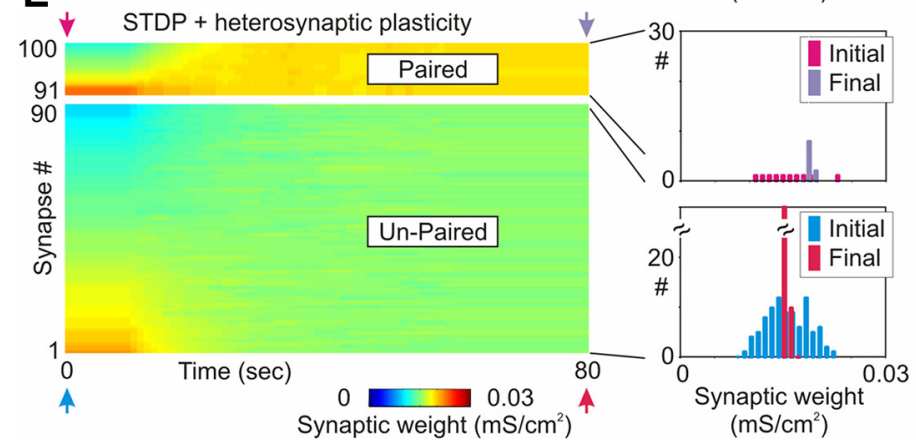

D
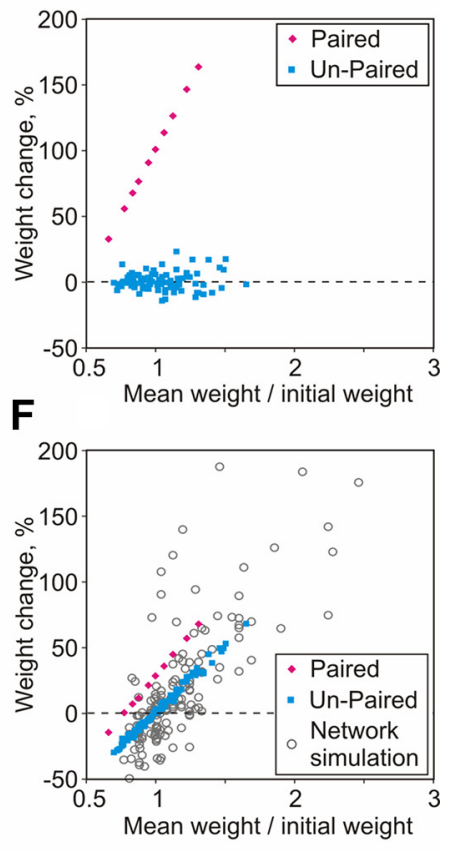

Figure 3. Homosynaptic and heterosynaptic plasticity in a model neuron. $\boldsymbol{A}$, A scheme of a model neuron receiving 100 synaptic inputs. $\boldsymbol{B}$, Rules for synaptic changes include homosynaptic STDP with dependence of the weight change on the timing between presynaptic and postsynaptic spikes (B1), and weight-dependent probability and weight change of heterosynaptic plasticity (B2; see Eqs. 9, 10). $\boldsymbol{C}, \boldsymbol{E}$, Dynamics of synaptic weight changes in the model with STDP-only rules $(\boldsymbol{C})$ and in the model with STDP and heterosynaptic plasticity $(\boldsymbol{E})$. In all simulations, a subset of inputs (synapses 91-100) was stimulated with a pairing protocol: synaptic activation was followed (after $10 \mathrm{~ms}$ ) by a burst of spikes induced by short depolarizing pulses to the soma. The pairing procedure was repeated 50 times, once per second. Remaining inputs to the neuron (synapses 1-90) were "unpaired" and were activated randomly with averaged frequency of $1 \mathrm{~Hz}$. Right, Weight distributions of synapses in the paired and unpaired groups, at the beginning (initial) and at the end (final) of the simulation. $\boldsymbol{D}, \boldsymbol{F}$, Correlation between synaptic weight changes and the ratio between the mean weight $\left(0.015 \mathrm{mS} / \mathrm{cm}^{2}\right)$ and initial weight of the synapse (this ratio is used as a proxy of the initial PPR measured in slice experiments), for paired and unpaired inputs from the simulations shown in $\boldsymbol{C}, \boldsymbol{E} . \boldsymbol{F}$, Gray circle symbols represent data for 150 synapses from a network simulation subject to different activity patterns (from Fig. 8C, $F$, a random sample of 150 synapses from $N=5324$ intrinsic synapses).

followed (with a small and controlled delay) by a burst of postsynaptic spikes induced by brief depolarizing pulses to the soma. At the same time, other synaptic inputs to the neuron were randomly active throughout the simulation with averaged frequency of $1 \mathrm{~Hz}$. In the model with STDP-only, paired inputs were rapidly potentiated to the maximal value and remained saturated until the end of the simulation (Fig. 3C; synapses 91-100). Unpaired inputs changed little (Fig. 3C; synapses 1-90). Although heterosynaptic plasticity was not implemented in this model, some of the unpaired inputs still changed, apparently because they were occasionally active within STDP windows of postsynaptic spikes (Benuskova and Abraham, 2007).

To compare weight dependence of synaptic changes in this model to the same results of slice experiments, we used the ratio of mean weight $\left(0.015 \mathrm{mS} / \mathrm{cm}^{2}\right)$ over the initial weight as a proxy of initial PPR measures in slice experiments (Fig. 2A). In the model, scatter plots of synaptic weight changes against the ratio of mean/initial synaptic weight revealed weight dependence of changes in paired inputs, but no weight dependence of changes in unpaired synapses (Fig. 3D). Absence of weight dependence of changes in unpaired inputs in the STDP-only model was in contrast to the results derived from slice experiments (Fig. 2A). Next, we applied exact same stimulation protocol (pairing of synapses 91-100 with bursts of postsynaptic spikes, and random activity in synapses 1-90) to a model in which both STDP and heterosynaptic plasticity were implemented. In this model, both paired and unpaired synaptic inputs changed their weights and formed clearly separate distributions; furthermore, no synapses were saturated (Fig. 3E). The plot of synaptic changes against the ratio of mean/initial weights revealed weight dependence of changes in both groups of synapses, with the data points representing paired inputs shifted upwards relative to the unpaired inputs (Fig. $3 F$ ). In this plot (Fig. 3F, gray), we also show data for 150 randomly selected intrinsic synapses (of total $N=5324$ intrinsic synapses in a network model) from a 100 neuron network model with rich intrinsic connectivity (described below; see Fig. 8). This network model was stimulated with diverse input patterns, leading to a substantial variability of synaptic changes, a scenario that is closer to the diversity of conditions experienced by real neurons. Simulation results with the model, including both STDP and heterosynaptic plasticity presented in Figure $3 F$, have a clear similarity to the results of slice experiments (Fig. 2A).

Thus, model simulations show that a model with both STDP and heterosynaptic plasticity reproduces experimental data bet- 

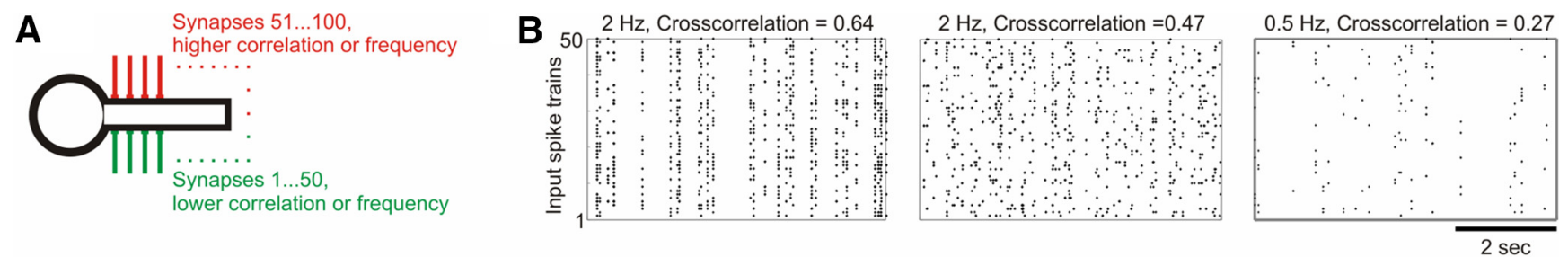

C

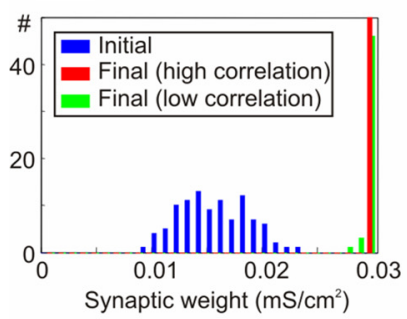

STDP only

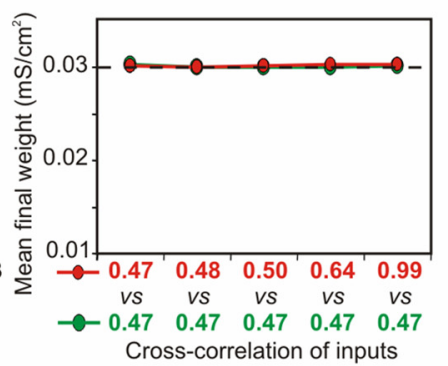

E

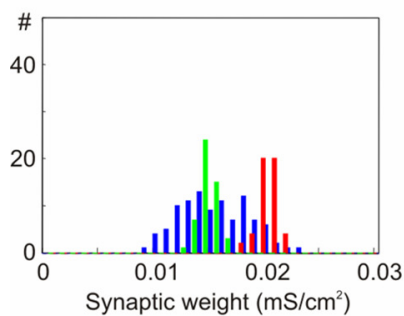

STDP + Heterosynaptic plasticity

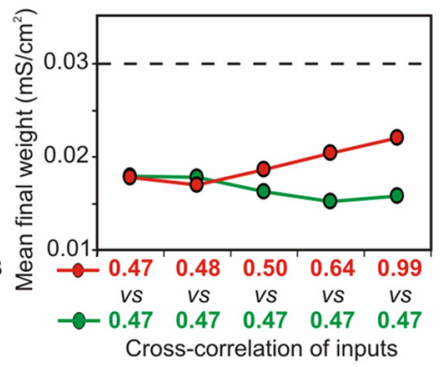

D

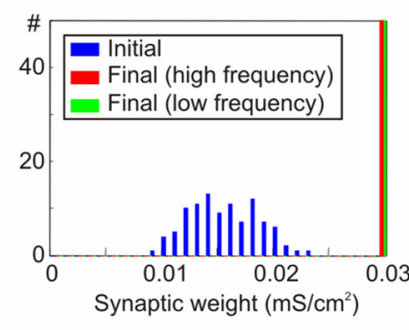

STDP only

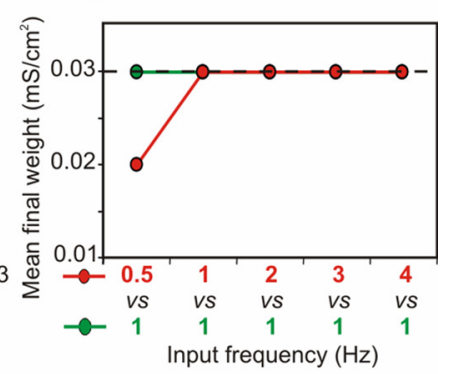

$\mathbf{F}$

STDP + Heterosynaptic plasticity
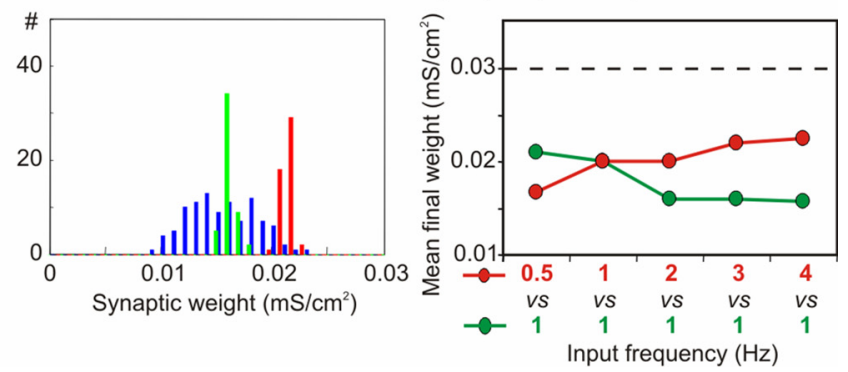

Figure 4. Heterosynaptic plasticity facilitates segregation of the weights of synapses subject to diverse presynaptic activity. $A, A$ scheme of the model neuron receiving two groups of synaptic inputs that differ in the level of correlation or rate of firing. $\boldsymbol{B}$, Examples of input patterns with different levels of correlation and averaged firing rates. $C-\boldsymbol{F}$, Left panels, Distributions of synaptic weights, initial (blue), and final after $200 \mathrm{~s}$ of simulation for synapses $1-50$ (green) and 51-100 (red). Two groups of inputs differed by the level of cross-correlation (0.64 vs 0.47 ) in $\boldsymbol{C}, \boldsymbol{E}$ and the averaged firing rate ( $3 \mathrm{~Hz}$ vs $1 \mathrm{~Hz}$ ) in $\boldsymbol{D}$, F. Right panels, Averaged final synaptic weight for the two groups of synapses, with input activity indicated below the plots. Models with the STDP only rule in $\boldsymbol{C}, \boldsymbol{D}$ and models with STDP and heterosynaptic plasticity in $\boldsymbol{E}, \boldsymbol{F}$.

ter than the STDP-only model. Further, these simulation results illustrate two basic points: (1) final distribution of synaptic weights depends on the interaction between homosynaptic and heterosynaptic plasticity; and (2) the model with both STDP and heterosynaptic plasticity supports segregation of the weights of synapses, which experienced distinct activity patterns.

\section{Heterosynaptic plasticity promotes differentiation of synaptic weights}

We next explored the role of heterosynaptic plasticity in synaptic competition and differentiation of synaptic weights. In these simulations, we did not use an explicit pairing protocol, but rather modeled a more realistic scenario in which synaptic activity induces action potentials in a neuron and may lead to synaptic plasticity. We modeled a neuron receiving two groups of synaptic inputs: "strongly" active, characterized by higher frequency or cross-correlation of presynaptic firing; and "weakly" active, with lower frequency or correlation of presynaptic firing (Fig. 4A,B). In the STDP-only model, most of the tested patterns of presynaptic firing induced runaway potentiation, so that both strongly and weakly active inputs acquired similar weights, typically saturated at the maximal weight (Fig. $4 C, D$ ). In contrast, in the model with both STDP and heterosynaptic plasticity, synaptic weights were not saturated but formed distinct distributions (Fig. 4E,F). Even a small difference in correlation level ( 0.47 vs 0.5$)$ or frequency of presynaptic firing $(0.5 \mathrm{vs} 1 \mathrm{~Hz}$, or $1 \mathrm{vs} 2 \mathrm{~Hz})$ between the two groups resulted in the divergence of the final synaptic weights. Importantly, the separation of the two distributions increased with the increasing difference in the level of correlation or rate of presynaptic firing (Fig. $4 E, F$, right panels). Thus, the model with both STDP and heterosynaptic plasticity was able to segregate weights of synapses experiencing different activity patterns, with the difference in the final weights proportional to the differences in the presynaptic activity.

Experimental evidence shows that plasticity rules may differ across synapses (Nishiyama et al., 2000; Sjöström et al., 2001; Zhou et al., 2005; Haas et al., 2006; Feldman, 2009) and that plastic abilities of synapses may change over time (Abraham and Bear, 1996; Abraham, 2008). Therefore, we next asked how dynamics of synaptic weight changes differ in two groups of synapses with different plasticity rules. In these simulations, the STDP rule was symmetrical in synapses of the first group but depression-biased in the second group (Fig. 5A). Synapses of both groups were activated with the same average frequency ( 3 $\mathrm{Hz}$ ) and correlation (0.5). In the STDP-only model, the difference in STDP rules caused runaway dynamics of synaptic weights to opposite extremes. As a result, the final steady-state weights of synapses with symmetrical STDP were saturated at the maximal value, and the weights of synapses with depression-biased STDP were all close to 0 (Fig. $5 B$ ). Addition of heterosynaptic plasticity to all synapses effectively prevented runaway dynamics and saturation but did not preclude segregation of synaptic weights. At the end of simulation, synapses with two different STDP rules formed two clearly distinct distributions within the operation 
A

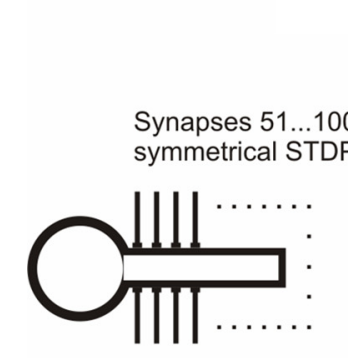

Synapses $1 \ldots 50$, depression-biased STDP

D
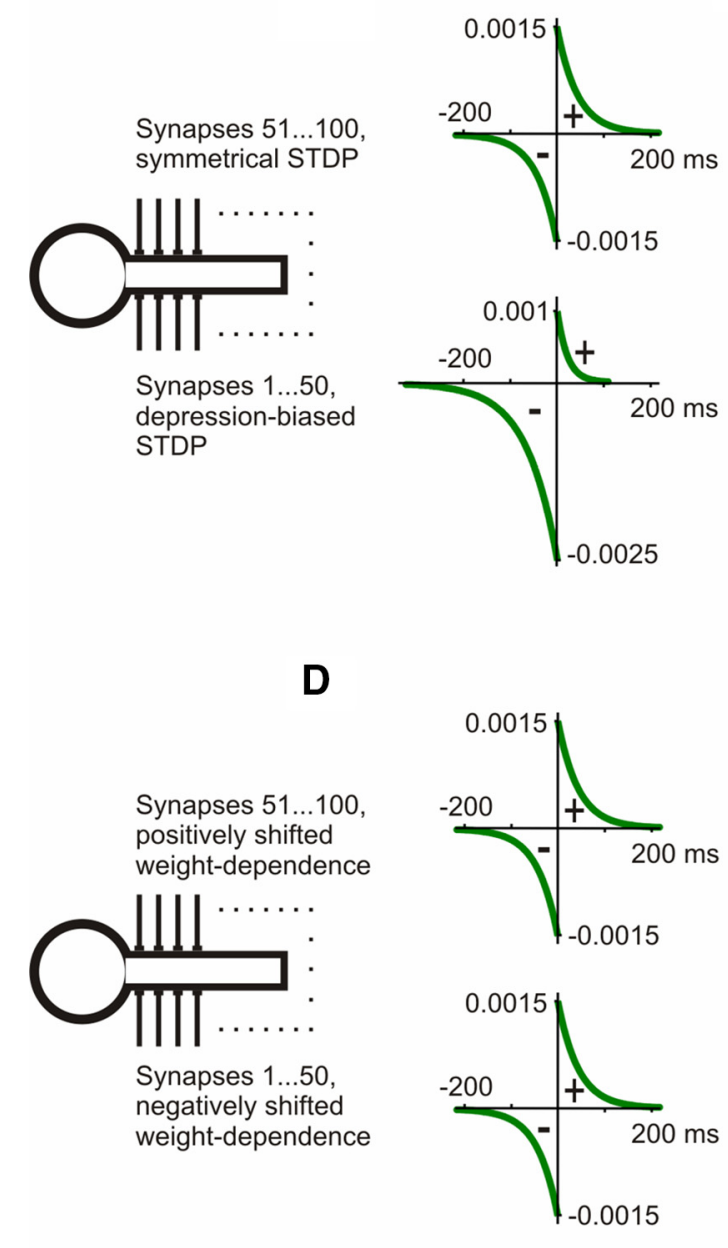

0.0025
B
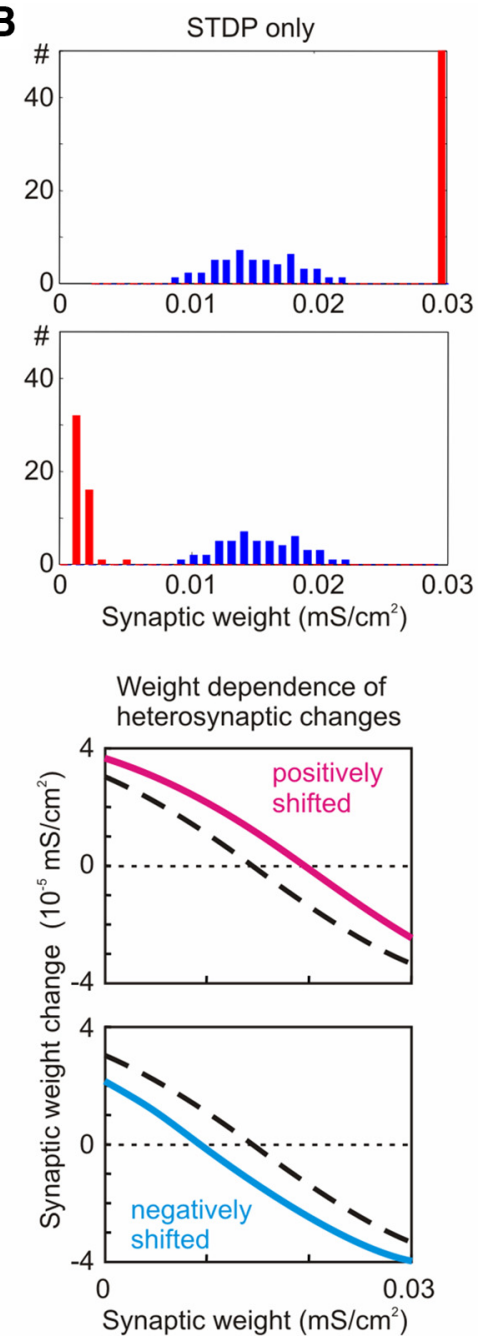

C
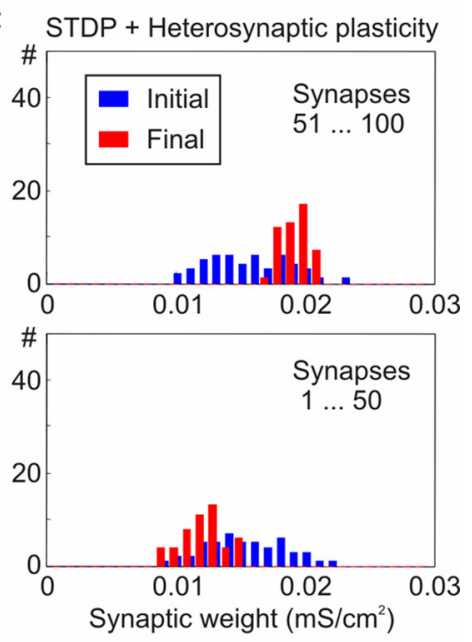

E
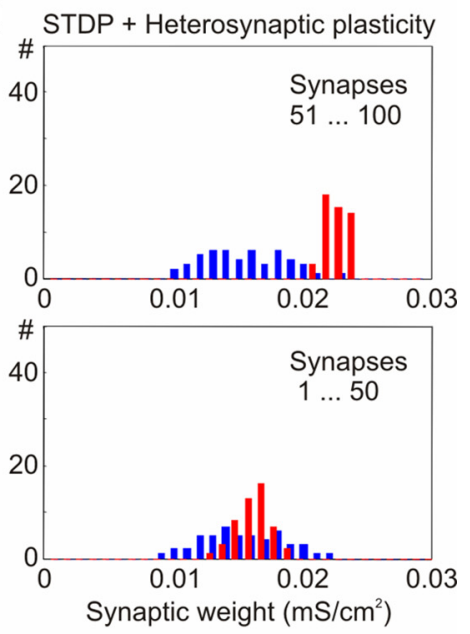

Figure 5. Segregation of synapses expressing different plasticity rules. $A$, Diverse STDP rules for two groups of synapses. STDP with symmetrical windows for potentiation and depression for synapses 51-100 ( $\left.\mathrm{a}^{+}=\mathrm{a}^{-}=0.0015 \mathrm{mS} / \mathrm{cm}^{2} ; \tau^{+}=\tau^{-}=40 \mathrm{~ms}\right)$, and depression-biased STDP for synapses $1-50\left(\mathrm{a}^{+}=0.001 \mathrm{mS} / \mathrm{cm}^{2} ; \tau^{+}=20 \mathrm{~ms} ; \mathrm{a}^{-}=0.0025 \mathrm{mS} / \mathrm{cm}^{2} ; \tau^{-}=60 \mathrm{~ms}\right)$. $\boldsymbol{B}, \boldsymbol{C}$, Distributions of synaptic weights of synapses $1-50$ and $51-100$ at the beginning (initial, blue) and after 100 s of simulation (final, red) in STDP-only model (B) and in the model with both STDP and heterosynaptic plasticity (C). D, The weight dependence of heterosynaptic changes was shifted right (positively shifted, magenta curve) for synapses $51-100$ and to the left (negatively shifted, cyan curve) for synapses 1-50. The STDP rule was the same in all synapses ( $\left.\mathrm{a}^{+}=\mathrm{a}^{-}=0.0015 \mathrm{mS} / \mathrm{cm}^{2} ; \tau^{+}=\tau^{-}=40 \mathrm{~ms}\right)$. , Initial and final distributions of synaptic weights in the model with symmetrical STDP and positively (top) or negatively (bottom) shifted weight dependence of heterosynaptic plasticity. $\boldsymbol{B}, \boldsymbol{C}, \boldsymbol{E}$, In all simulations, averaged rate of presynaptic firing was $3 \mathrm{~Hz}$ and averaged cross-correlation between spike trains was 0.5 .

range, with significantly different mean values $(0.0193 \pm 0.001$ $\mathrm{mS} / \mathrm{cm}^{2}$ vs $0.0123 \pm 0.0016 \mathrm{mS} / \mathrm{cm}^{2}, p<0.001$; Fig. $\left.5 C\right)$. In the model without heterosynaptic plasticity, but with weightdependent probability of STDP, runaway dynamics were not prevented, demonstrating that weight dependence of the probability of synaptic changes alone is not sufficient for preventing runaway dynamics. Addition of weight dependence of the potentiation in the STDP rule (magnitude of potentiation window decreases for stronger synapses, whereas depression window remains the same for all synaptic weights) (van Rossum et al., 2000) could prevent runaway dynamics. However, this solution was sensitive to specifics of STDP rules and weight dependence of potentiation, and the model required fine-tuning to be stable (van Rossum et al., 2000). Moreover, the weight dependence of STDP model was able to segregate the weights of synapses receiving different input activity patterns, without saturating the weights, in only a restricted range of STDP rules and input patterns (data not shown). In contrast, models with heterosynaptic plasticity robustly segregated, but not saturated, synaptic weights for a broad range of plasticity rules, input correlations, and rates.
In the above simulations (Fig. $5 C$ ), the rules of heterosynaptic plasticity were the same for all synapses. Will synapses governed by different rules of heterosynaptic plasticity still be segregated? To address this question, we used the same STDP rules in all synapses (Fig. 5D, left) but shifted the weight dependence of heterosynaptic plasticity (see Eq. 10) to the right in one group of synapses, and to the left in the other group (Fig. 5D, magenta and cyan curves). Final equilibrium weights of the two groups of synapses formed clearly separable distributions with significantly different mean values $\left(0.0163 \pm 0.0012 \mathrm{mS} / \mathrm{cm}^{2}\right.$ vs $0.0228 \pm 0.00083 \mathrm{mS} / \mathrm{cm}^{2}, p<$ 0.001 ; Fig. $5 E)$.

We concluded that synapses that experience different patterns of presynaptic activity, or have differential abilities for homosynaptic or heterosynaptic plasticity, can segregate their weights. The final distribution of synaptic weights, as well as the final weight of an individual synapse, are determined by the competing driving forces introduced by the rules of homosynaptic and heterosynaptic plasticity (Fig. 5) and/or patterns of presynaptic and postsynaptic activity (Figs. 3, 4). 
A1

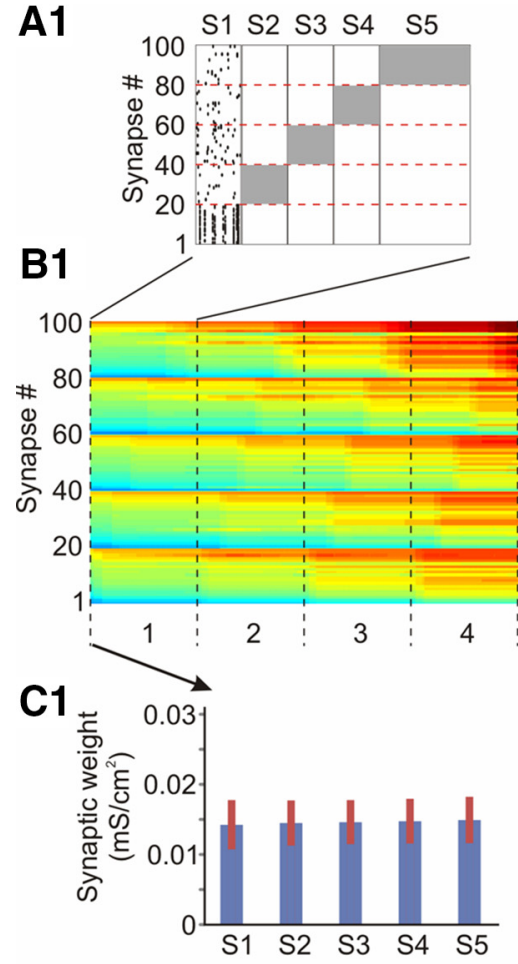

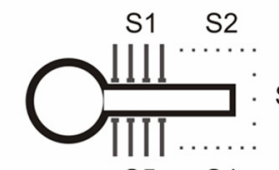

S5 S4
S3

STDP only
D1

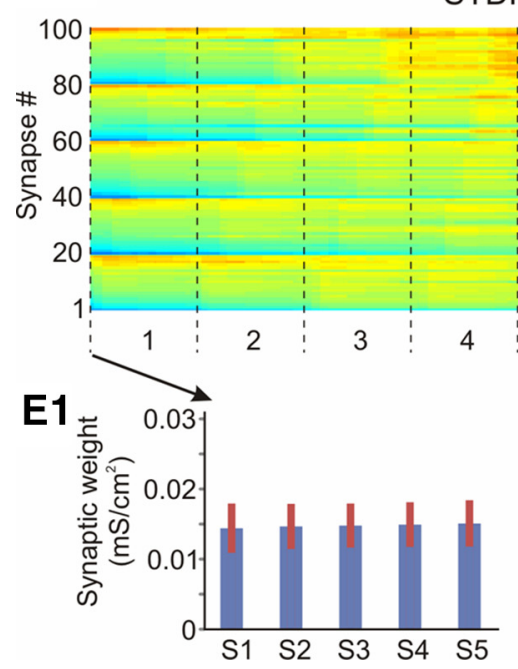

STDP + Heterosynaptic plasticity

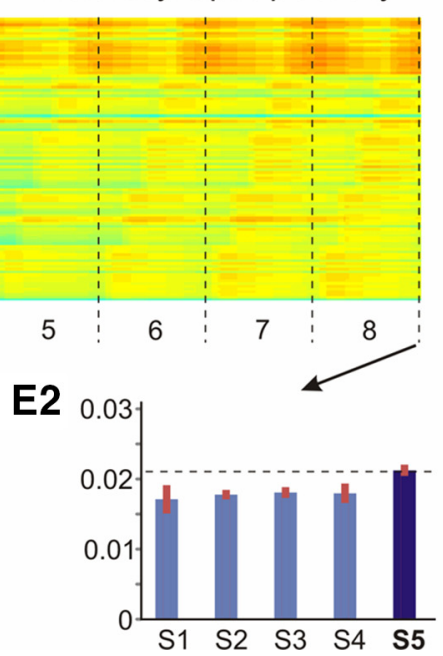

A2

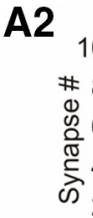

\section{B2}
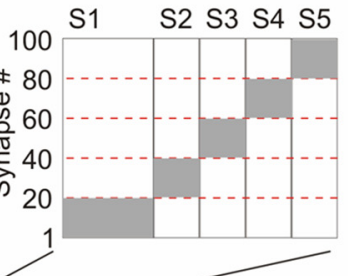
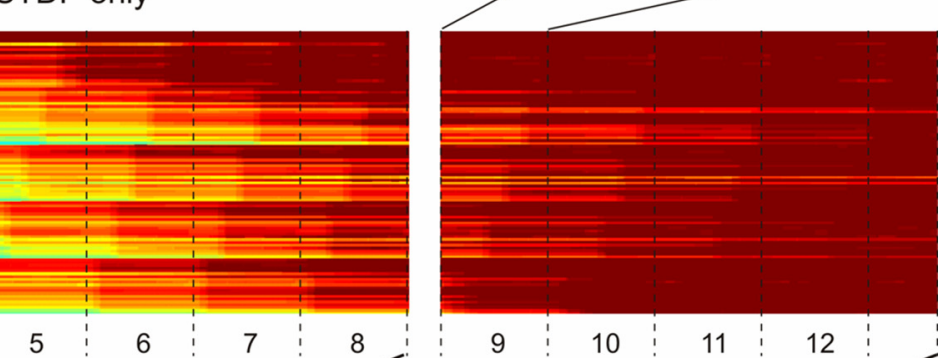

C3

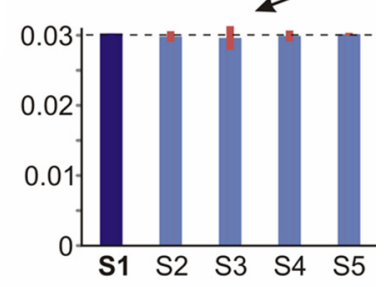

D2

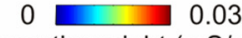
Synaptic weight $\left(\mathrm{mS} / \mathrm{cm}^{2}\right)$

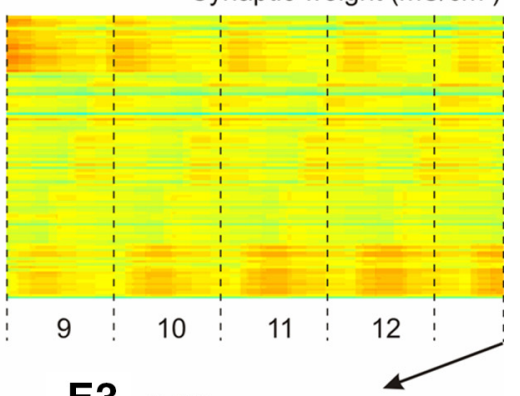

E3

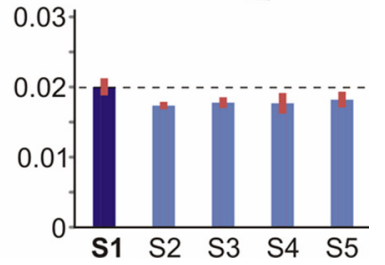

Figure 6. Heterosynaptic plasticity helps to extract the most frequently presented stimulus and allows relearning. $A$, Blocks of five "stimuli," each stimulus defined by higher rate $(4 \mathrm{~Hz})$ and correlation $(0.9 \pm 0.01)$ of firing in 20 of 100 inputs. Spikes in the remaining 80 inputs occurred at $1 \mathrm{~Hz}$, with correlation of $0.34 \pm 0.02$. During the first training session, stimuli S1-S4 were presented for $600 \mathrm{~ms}$, and S5 for $1200 \mathrm{~ms}$ (A1), in each of 8 blocks. In the second session, S1 was presented for $1200 \mathrm{~ms}$ and S2-S5 for $600 \mathrm{~ms}$ in each block (A2). Inset, Scheme of model neuron receiving input stimuli S1-S5. $\boldsymbol{B}$, Weight changes of 100 synapses with STDP-only rules $\left(\mathrm{a}^{+}=\mathrm{a}^{-}=0.001 \mathrm{mS} / \mathrm{cm}^{2} ; \tau^{+}=\tau^{-}=20 \mathrm{~ms}\right)$ during two training sessions. $\boldsymbol{B}$, In the first session, eight blocks of stimuli with more frequent $S 5$ (as in $\boldsymbol{A}$ ) were presented. B2, In the second session, four blocks of stimuli with more frequent $S 1$ (as in $A 2$ ) were presented. $\boldsymbol{C}$, Averaged weights ( \pm SD) of synapses representing stimuli S1-S5 before learning ( $(1)$, after the first training session (C2) (one-way ANOVA: $p=0.0052$ difference between groups, and $p=0.023, p=0.036, p=0.024, p=$ 0.008 for comparisons Group 5 vs Groups 1-4, respectively), and after the second session (C3) (one-way ANOVA: $p=0.175$ for difference between groups, and $p>0.1$ for Group 1 vs Groups $2-5$, respectively). Dashed lines indicate averaged weights of synapses representing more frequent stimulus ( $S 5$ in $C 2$ and $S 1$ in C3).D, E, Synaptic weight changes and mean synaptic weights in the model with STDP and heterosynaptic plasticity, before (E1), after (E2) the first training session (D1) $(p<0.001$ for difference between groups, and for comparisons of Group 5 vs Groups $1-4)$, and after (E3) the second training session (D2) ( $p<0.001$ for difference between groups, and for comparisons of Group 1 vs Groups $2-5)$. Same stimuli and same representation as in $\boldsymbol{B}, \boldsymbol{C}$.

\section{Heterosynaptic plasticity helps to extract the most frequently presented pattern}

In the previous sections, we tested models with stimulation patterns that remain unchanged throughout the simulations. In real-life scenarios, activity changes over time, such that some patterns or combinations of stimuli are presented more often than others. To study dynamics of synaptic weights and activity in model neurons in such a realistic setting, we divided synaptic inputs to a model neuron into 5 groups (20 synapses in each group) representing 5 different "stimuli," S1-S5 (Fig. 6). A stimulus was defined by stronger activity within a selected group of 20 synaptic inputs (averaged firing rate $4 \mathrm{~Hz}$, correlation 0.91 ) on the background of weak activity in the remaining 80 inputs $(1 \mathrm{~Hz}$, correlation 0.34 ). Stimuli were presented in repeated blocks. In 
A
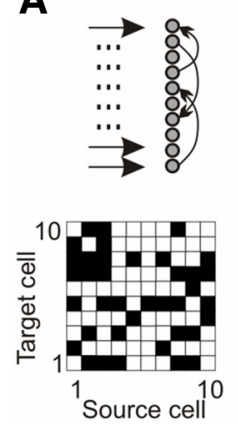

B
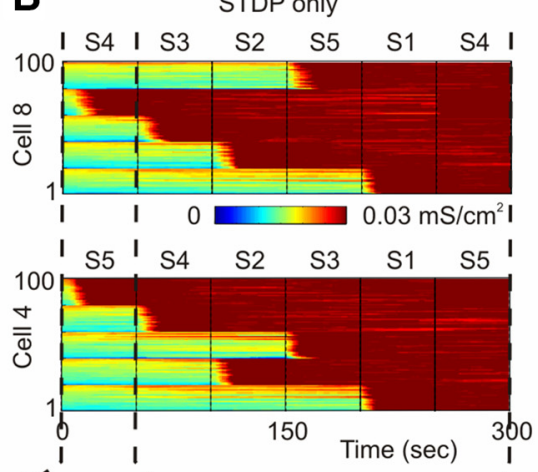

C

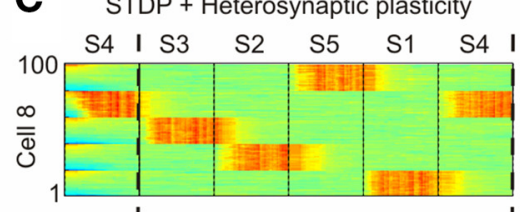

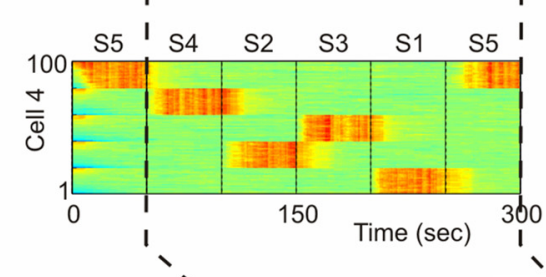

D - STDP only - STDP + Heterosyn

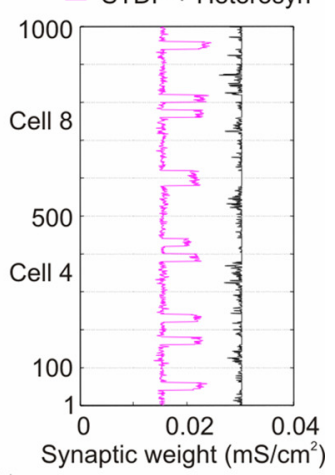

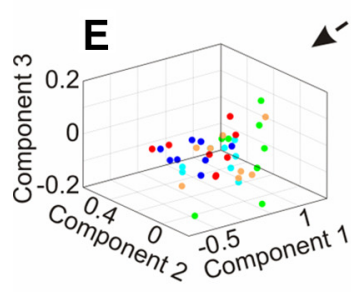
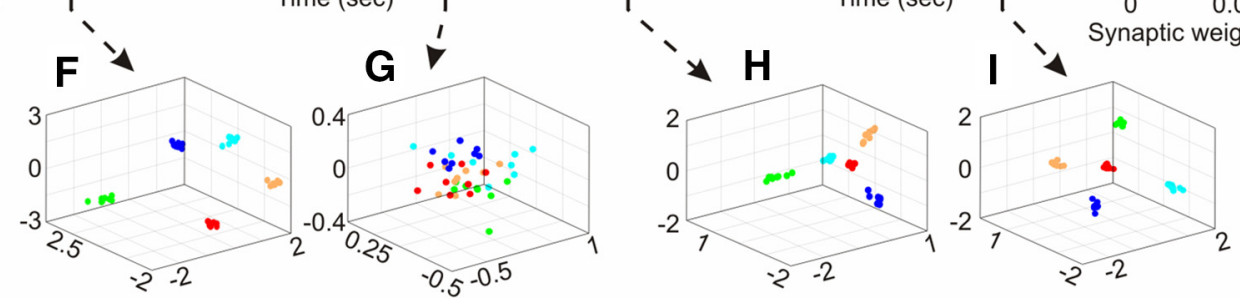

- $\mathrm{S1}$

- $\mathrm{S} 2$

- 53

- S4

- S5

Figure 7. Heterosynaptic plasticity allows relearning and segregation of learned patterns in a small network. $A, A$ scheme of a simple network of 10 neurons. Each neuron receives 100 external inputs organized in 5 groups of 20 (S1-S5, as in Fig. 6 A, inset), and is connected to other neurons in the network with probability $p=0.5$, as shown in connectivity matrix. $\boldsymbol{B}, \boldsymbol{C}$, Dynamics of synaptic weights in 2 sample cells of the network during stimulation with different patterns of activity, as indicated. Each neuron received an individual, pseudo-random sequence of stimuli. Model with STDP-only plasticity rules (B), and model with STDP and heterosynaptic plasticity (C). D, Synaptic weights in the 10 neurons of the network at the end of simulation, in the STDP-only model (black) and in the model with STDP and heterosynaptic plasticity (magenta) learning rules. Dashed horizontal lines separate synapses to different neurons, synapses of cells 4 and 8 from $\boldsymbol{B}, \boldsymbol{C}$ are indicated. Only external inputs are shown here. Internal inputs were also plastic; but because of their low number, they did not contribute significantly to postsynaptic firing and are not shown. $\boldsymbol{E}-\boldsymbol{I}$, Principal component analysis of responses of the network to test stimuli S1-S5, at the beginning of the simulation (E), and after the first $(\boldsymbol{F})$ and sixth $(\boldsymbol{G})$ episodes of training in STDP-only model, and in the model with STDP and heterosynaptic plasticity $(\boldsymbol{H}, \boldsymbol{I})$. There is scale change between plots.

each block, stimuli S1-S4 were presented for $600 \mathrm{~ms}$ each, followed by a longer (1200 ms) presentation of S5 (Fig. 6A1).

In the STDP-only model, repeated presentation of the blocks of stimuli led to potentiation of all synapses, whereby the weights of synapses representing most frequent stimulus (S5, synapses 80-100 in Fig. 6B1) increased faster. The changes of synaptic weights first remained selective (see results of training with 8 blocks of stimuli in Fig. 6C2; one-way ANOVA, $p=0.0052$ difference between groups, and $p=0.023, p=0.036, p=0.024, p=$ 0.008 for comparisons Group 5 vs Groups 1-4, respectively). However, this state was not stable: after presentation of additional stimulation blocks, synapses representing any stimulus, both frequently and less frequently presented, became saturated and segregation of synaptic weights effectively disappeared (data not shown; $p>0.1$ for all comparisons).

In contrast, the model equipped with both STDP and heterosynaptic plasticity was able to robustly segregate a group of synapses representing the most frequent stimulus. These synapses became significantly stronger than synapses representing other stimuli after only $4-5$ blocks of stimulation (Fig. $6 D 1$ ). This difference remained significant for steady-state weight distributions (Fig. 6E2; one-way ANOVA, $p<0.001$ for difference between groups, and for comparisons of Group 5 vs Groups 1-4), and importantly, was preserved after further presentations of the same blocks of stimuli (data not shown).

\section{Heterosynaptic plasticity allows relearning}

Advantages of the model with both STDP and heterosynaptic plasticity over the model with STDP-only rules became especially clear in a paradigm of relearning. After presenting 8 blocks of stimuli as described above, we ran a second training session, but with an altered composition of the stimuli in the block so that a different stimulus was presented for a longer time (S1 for 1200 ms, followed by S2-S5, each for 600 ms; Fig. 6A2). In the STDPonly model, synaptic weights continued their trend toward the maximal value, and most of the synapses were saturated after presentation of 4 blocks of stimuli in the second training session (Fig. 6B2,C3; one-way ANOVA, $p=0.175$ for difference between groups, and $p>0.1$ for comparisons of Group 1 vs Groups 2-5). In contrast, in the model with STDP and heterosynaptic plasticity, the change of the stimulus configuration transformed the dynamics of synaptic weights. The weights of synapses representing the new most-frequent stimulus (S1) increased, whereas weights of synapses representing the previously learned stimulus (S5) decreased (Fig. 6D2). After presentation of 4 blocks, a new representation was built: synapses representing the new frequent stimulus ( $\mathrm{S} 1$ ) acquired significantly higher weights than synapses representing other stimuli (Fig. 6E3; one-way ANOVA, $p<0.001$ for difference between groups, and for comparisons of Group $1 \mathrm{vs}$ Groups 2-5).

\section{Heterosynaptic plasticity prevents runaway dynamics and allows relearning in a neuronal network}

Having established the role of heterosynaptic plasticity in singleneuron models, we next asked how heterosynaptic plasticity affects dynamics of synaptic weights, evolution of activity, and learning abilities in model networks.

We trained a generic network consisting of 10 identical neurons (Fig. 7A), with a pattern of 5 stimuli (S1-S5; see description above), so that each specific stimulus was presented to 2 neurons. During the first episode of training, synaptic weights changed in both the STDP-only model and the model with both STDP and heterosynaptic plasticity, until reaching a steady state (Fig. $7 \mathrm{~B}, \mathrm{C}$; $0-50 \mathrm{~s})$. Principal component analysis revealed that both models built distinct representations of stimuli S1-S5, which could be clearly discriminated from responses of the model neurons 
A

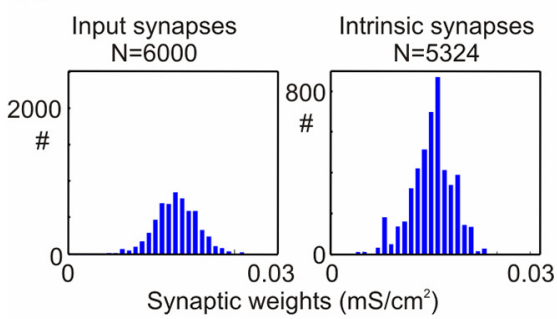

B

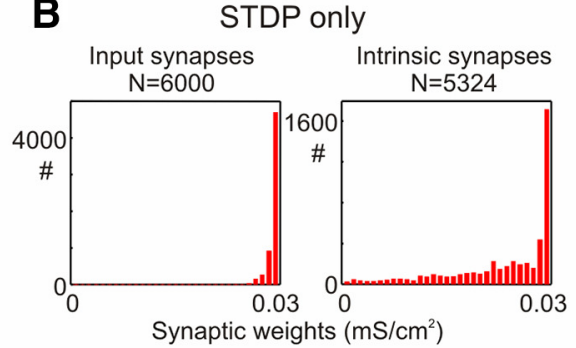

C STDP + Heterosynaptic plasticity

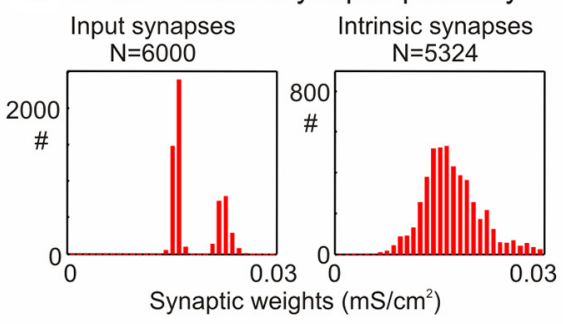

D

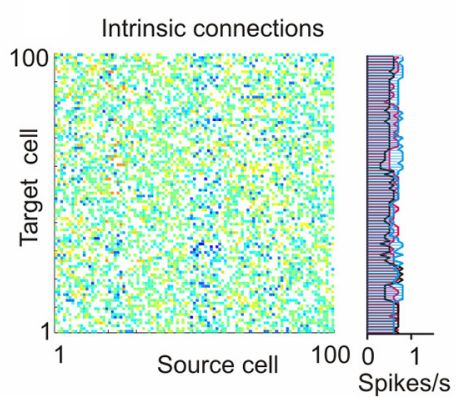

E

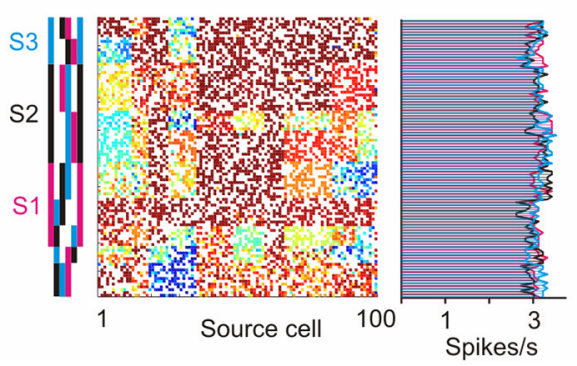

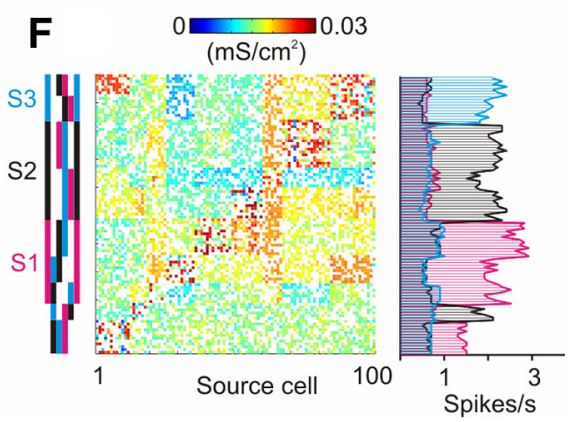

Figure 8. Heterosynaptic plasticity facilitates formation of connectivity patterns in a generic neuronal network. The generic network consisted of 100 neurons, each receiving 60 external inputs (3 groups of 20 synapses representing S1-S3), and intrinsic synapses from other network neurons, with $p=0.5$ connection probability. Each stimulus $\$ 1-\$ 3$ was uniquely represented by higher average frequency $(4 \mathrm{~Hz}$ ) and correlation (0.91) of input spike trains for one group of synapses compared with the background activity at the other inputs (1 $\mathrm{Hz}$, averaged correlation 0.34$)$. $\boldsymbol{A}-\boldsymbol{C}$, Distributions of weights of input synapses to all neurons of the network $(N=6000$ synapses to 100 neurons), and all intrinsic synapses $(N=5324$ synapses $)$ at the beginning of simulations $(\boldsymbol{A})$, after training in the STDP-only model $(\boldsymbol{B})$, and in the model with STDP and heterosynaptic plasticity (C). $\boldsymbol{D}-\boldsymbol{F}$, Matrix of intrinsic connections in the network, with color-coded synaptic weights: before training $(\boldsymbol{D})$, after training in the STDP-only model $(\boldsymbol{E})$, and in the model with STDP and heterosynaptic plasticity $(\boldsymbol{F})$. The training consisted of 6 episodes, 50 s each, with input pattern changing between the episodes. Pattern of presentation during training is shown in $\boldsymbol{E}, \boldsymbol{F}$ (left; the same pattern of presentation in these two simulation tests; magenta represents $\mathrm{S1}$; black represents $\mathbf{S 2}$; cyan represents S3; white represents background). Before and after training, each stimulus was presented to all neurons of the network for $10 \mathrm{~s}$, and averaged spiking frequency of response to each stimulus was calculated and plotted for each neuron ( $D-F$, right; magenta represents S1; black represents S2; cyan represents S3).

(compare Fig. $7 E$ with Fig. $7 F, H$ ). However, continuing training with new input patterns (the same stimuli S1-S5 presented to neurons in a different combination; see description above), revealed a fundamental difference between two models in the dynamics of synaptic weights and learning. In the STDP-only model, training with each new pattern led to strengthening of an additional set of synapses. By the end of simulation all synapses in STDP-only model were at or close to the maximal value (Fig. $7 B$ ). Distribution of synaptic weights did not reflect any distinct input pattern (Fig. 7D, black line), and responses of STDP-only model to different stimuli became indistinguishable (Fig. $7 G$ ). In the model with STDP and heterosynaptic plasticity, synaptic weights were redistributed after each change of the input pattern, such that distribution of synaptic weights represented the most recently trained stimulus (Fig. 7C). By the end of simulation, synapses of the model with STDP and heterosynaptic plasticity were not saturated. The last trained pattern was represented in synaptic weights (Fig. 7D, magenta line), and trained stimuli could be discriminated from responses of the network model with STDP and heterosynaptic plasticity (Fig. 7I).

These results show that the addition of heterosynaptic plasticity allows neuronal networks to form reliable and distinct representations of trained input patterns and rebuild these representations according to changing experience. This provides networks with the ability to keep the configuration of synaptic weights up-to-date with changing inputs and respond differentially to distinct stimuli.

\section{Heterosynaptic plasticity promotes development of input- related connectivity and response selectivity in networks of interconnected neurons}

To study the dynamics of synaptic weights and activity in a network with strong intrinsic connectivity, we increased the number of neurons in the network to 100 . Each neuron received $\sim 50$ (range 37-57) intrinsic synapses from other neurons (connection probability $=0.5)$, and 60 external synapses, organized in three groups of 20 synapses representing 3 stimuli (S1-S3). During one training episode, each neuron received external input representing one of the stimuli S1-S3 or background activity. The training consisted of 6 episodes, $50 \mathrm{~s}$ each, with the input pattern changing between the episodes.

In the STDP-only model, each episode of training led to runaway potentiation of external synapses corresponding to the trained stimulus. The weights reached their maximum and changed little after that, even when a different input pattern was presented. By the end of all training episodes, all input synapses were potentiated to the maximal value or close to it (Fig. 8B, left). Additionally, intrinsic connections between neurons achieved maximal, or close to maximal weights (Fig. $8 B$, right). Predominance of potentiation and saturation of intrinsic synapses in the network with STDP-only plastic rules is clearly demonstrated by the matrix of intrinsic network connections, with color-coded synaptic weights (Fig. $8 D, E$ ).

In contrast to the runaway potentiation in the STDP-only model, the evolution of synaptic weights in the same network but equipped with STDP and heterosynaptic plasticity learning rules revealed balanced dynamics (Fig. $8 C, F$ ). Input synapses changed according to the stimulation pattern, with synapses representing the stimulus acquiring the larger weights. Importantly, this representation was dynamic and adjustable: changing of the training pattern led to redistribution of the weights of input synapses forming a new representation. At the end of training, the weights of input synapses had a bimodal distribution, where stronger synapses represented the stimuli delivered during the most recent training episode (Fig. 8C, left). Intrinsic synapses between neu- 
rons of the network did not saturate, either. Their weight distribution displayed a tail of stronger weights that were not present initially (Fig. 8C, right). Analysis of the stimulation history revealed that synapses between neurons, which experienced similar stimulation acquired stronger weights (Fig. $8 F$ ). This is remarkable because, although intrinsic synapses were not explicitly activated by the input stimuli, the emerged pattern of stronger connections reflects common "sensory" experience of neurons.

Training led to the development of profoundly different response properties in the networks with or without heterosynaptic plasticity. Before training, the naive network responded similarly to all three stimuli (Fig. 8D, right). After training, responses of the STDP-only model increased several-fold but remained nonselective (Fig. 8E, right). In contrast, the network with STDP and heterosynaptic plasticity developed selective responses after the same training. Each neuron responded stronger to one (last-trained) stimulus. Notably, neurons that experienced background activity during the last training episode(s), responded selectively to the stimulus presented in the previous training episode (S2, cells 13-18) or an episode before it (S1, cells 1-12; Fig. 8F).

\section{Discussion}

We report that heterosynaptic changes of nonactivated synapses accompany the induction of STDP in neocortical neurons. Hence, the postulate of input specificity of STDP, while holding true on average, breaks down at the level of individual synapses. We characterize, for the first time, key properties of this novel form of heterosynaptic plasticity: weight dependence and balanced nature of potentiation and depression. We further show that implementing this novel form of heterosynaptic plasticity in model neurons and networks endows the models with essential computational features. It effectively prevents runaway dynamics of synaptic weights and activity, promotes segregation of synaptic weights and synaptic competition, and enables relearning and acquisition of new memories. Moreover, heterosynaptic plasticity promotes the development of selectivity of neuronal responses and intrinsic connections in generic networks of interconnected neurons.

\section{Heterosynaptic changes accompany induction of STDP in neocortical neurons}

We show that a pre-before-post STDP protocol leads, along with homosynaptic potentiation, to heterosynaptic plasticity in unpaired synapses. Heterosynaptic changes were (1) weight-dependent; their direction and magnitude correlated with initial PPR; and (2) balanced; despite significant potentiation or depression of individual synapses, EPSP amplitudes averaged over all unpaired inputs $(N=71)$ were not significantly different from the control period. These results constitute an important discovery that weight-dependent and balanced heterosynaptic changes can be induced not only by purely postsynaptic protocols, such as bursts of spikes without presynaptic activation, as reported previously (Volgushev et al., 1997, 2000; Chistiakova and Volgushev, 2009; Lee et al., 2012; Chen et al., 2013), but also by conventional STDP protocols.

Heterosynaptic changes apparently contradict the notion of input specificity (Bliss and Collingridge, 1993), which maintains that plasticity occurs only at synapses activated during the induction, whereas nonactive synapses should not change. Our results suggest that heterosynaptic changes often remain unnoticed because they are bidirectional, and LTP and LTD occurring in individual synapses balance each other. Thus, the postulate of input specificity, while holding true when heterosynaptic changes over many synapses are pooled together, breaks down at the level of individual synapses. This predicts that heterosynaptic changes would have high variance. Published data support this conjecture. Indeed, the variance of EPSP amplitude changes after postsynaptic spike bursts without presynaptic stimulation was reported to be very high, e.g., $93 \pm 39.7 \%$ (mean $\pm \mathrm{SD}, N=7$ ) (Birtoli and Ulrich, 2004) and $105 \pm 49 \%(N=6)$ (Birtoli and Ulrich, 2004; Nevian and Sakmann, 2006). To explain such high variance, there must have been significant LTP and LTD in individual synapses (for review, see Fiete et al., 2010; Chistiakova et al., 2014). The variance of synaptic changes in experiments with plasticity induction protocols involving postsynaptic spiking was significantly higher than that found over similar time periods in experiments where postsynaptic spiking was not induced (Volgushev et al., 2000). We conclude that heterosynaptic changes depend on the postsynaptic firing and accompany the induction of homosynaptic plasticity, and thus represent an inherent property of plastic synapses.

\section{Heterosynaptic plasticity endows model neurons with essential computational features}

Heterosynaptic plasticity with experimentally observed properties (induction at nonactive synapses, weight dependence of the direction and magnitude, and balanced potentiation and depression) introduces two essential computational properties to the neural systems with plastic synapses: (1) it has a normalizing effect on synaptic weights; and (2) it promotes synaptic competition. Functional consequences of these properties include prevention of runaway dynamics of synaptic weights and activity, enhanced abilities for learning and relearning, and enhanced segregation of inputs and intrinsic connections in neuronal networks.

The normalizing effect of heterosynaptic plasticity on synaptic weights is due to the weight dependence of synaptic changes: Strong synapses tend to depress, whereas weak synapses tend to potentiate. This drives synaptic weights away from extreme values, toward an equilibrium point within the operational range. This effect of heterosynaptic plasticity is different from a formal mathematical normalization. Mathematical normalization preserves total sum of synaptic weights and thus total synaptic drive to a cell but does not prevent runaway potentiation or depression of individual synaptic weights. Indeed, training in the models implementing normalization often leads to runaway dynamics of individual synaptic weights resulting in their bimodal distribution, with the "winner" synapses bunched around the maximal weight and the other synapses gathered around 0 (Song et al., 2000; van Rossum et al., 2000; Gütig et al., 2003; Morrison et al., 2007). In contrast, in our model implementing this novel form of heterosynaptic plasticity, individual synapses do not express runaway dynamics, and the weights of all synapses remain within the operation range. An important consequence of unsaturated weights is the ability for further learning. Indeed, our results demonstrate that models equipped with both STDP and heterosynaptic plasticity are able to relearn and to update their synaptic weights in accordance with changes of the external inputs.

The enhancement of synaptic weight segregation and synaptic competition by heterosynaptic plasticity can be understood as follows. For activated synapses, homosynaptic potentiation or depression prevails, pushing their weights toward the maximum or minimum. Heterosynaptic plasticity, triggered by the same episodes of activity that induce homosynaptic changes, imposes background forces that drive the weights of inactive synapses away from the extremes, toward an equilibrium value. As a result, changes of active versus inactive synapses are driven by contrasting forces, and have different target weights. In this scenario, 
synapses compete for maintaining their weights at the level set by homosynaptic plasticity but will be driven to the heterosynaptic equilibrium when other synapses cause postsynaptic spiking (Chistiakova et al., 2014, 2015). Modeling results from the present study provide evidence for such a scenario and show that, in models equipped with STDP and heterosynaptic plasticity, the segregation of synaptic weights can be caused by a broad range of factors, including frequency and correlation of presynaptic firing and details of the plasticity rules. This predicts that synaptic weights will be distributed broadly over the operation range rather than near extreme values. Experimental evidence supports this prediction (Sjöström et al., 2001; Song et al., 2005; Hardingham et al., 2007).

Through preventing the saturation of synaptic weights and promoting synaptic competition, heterosynaptic plasticity provides neurons and neuronal networks with the ability for continued learning and building dynamic representations of changing environments. In studies of decision making (Skorheim et al., 2014a) and auditory processing (Skorheim et al., 2014b), a model trained by the sequence of inputs reached synaptic weight saturation quickly and lost its ability for further learning unless heterosynaptic plasticity-like homeostatic rules were implemented. Our results show that, in networks of interconnected neurons, heterosynaptic plasticity is necessary for formation and refinement of intrinsic connectivity and development of response selectivity. This is consistent with results of a recent study, which showed that orchestrated action of multiple forms of plasticity, including heterosynaptic plasticity, is necessary for formation of assemblies in a model of a recurrent spiking network (Zenke et al., 2015). We conclude that heterosynaptic plasticity may play a role in maintaining the ability for new learning in a broad range of learning tasks and developmental processes.

The need for fast-scale mechanism(s) which prevent runaway dynamics of activity and support synaptic competition has been long appreciated in theoretical and modeling studies (von der Malsburg, 1973; Oja, 1982; K. M. Miller, 1994; K. D. Miller, 1996). A number of candidate homosynaptic mechanisms were suggested, including fine-tuning of STDP rules (Song et al., 2000; Kempter et al., 2001; Gütig et al., 2003; Babadi and Abbott, 2010) and activity dependence (Abraham and Bear, 1996; Yeung et al., 2004; Clem et al., 2008) or weight dependence of plastic rules (Bi and Poo, 1998; van Rossum et al., 2000; Hardingham et al., 2007). Other suggested heterosynaptic mechanisms include competition for resources (Frey and Morris, 1997, 1998; van Ooyen, 2001; Elliott and Shadbolt, 2002; Fonseca et al., 2004), or locally balanced potentiation and depression (Royer and Paré, 2003). In model simulations, stabilization of activity and synaptic competition can be attained by normalization implemented directly into the equations for synaptic weight changes (von der Malsburg, 1973; Oja, 1982; K. M. Miller, 1994; K. D. Miller, 1996; Fiete et al., 2010; Zenke et al., 2013). Our study suggests a novel physiological mechanism for solving these problems and mediating the vital theoretically predicted features of plastic synapses. Heterosynaptic plasticity with experimentally observed features introduces robust stability and synaptic competition as inherent properties of neurons with plastic synapses. It keeps synaptic weights in operation range, promotes synaptic competition, and endows learning neurons and networks with essential computational properties, allowing them to build and relearn representations of input stimuli. We conclude that the ability to undergo heterosynaptic changes is an inherent and indispensable feature of plastic synapses.

\section{Outlook}

The weight of a plastic synapse is determined by multiple factors: the patterns of presynaptic and postsynaptic activity, and the interaction between diverse mechanisms mediating homosynaptic and heterosynaptic plasticity. What are the rules of interaction between different forms of plasticity? How do these rules depend on changes of neuromodulatory background and on the related alterations of brain state? It is tempting to speculate that modulation of the balance between homosynaptic and heterosynaptic plasticity may underlie state-specific changes of the learning abilities.

Brain networks are built of morphologically and electrophysiologically diverse neurons, playing distinct roles in neuronal computations. What are type-specific rules and what are common features for homosynaptic and heterosynaptic plasticity across neurons? How do these plasticity rules tune neurons for serving their functions? Answering these questions is indispensable for building realistic models of neocortical networks and for understanding how the interplay of different forms of plasticity brings about the ability of neocortex to support life-long learning.

\section{References}

Abraham WC (2008) Metaplasticity: tuning synapses and networks for plasticity. Nat Rev Neurosci 9:387. CrossRef Medline

Abraham WC, Bear MF (1996) Metaplasticity: the plasticity of synaptic plasticity. Trends Neurosci 19:126-130. CrossRef Medline

Abraham WC, Goddard GV (1983) Asymmetric relationships between homosynaptic long-term potentiation and heterosynaptic long-term depression. Nature 305:717-719. CrossRef Medline

Babadi B, Abbott LF (2010) Intrinsic stability of temporally shifted spiketiming dependent plasticity. PLoS Comput Biol 6:e1000961. CrossRef Medline

Bazhenov M, Timofeev I, Steriade M, Sejnowski TJ (2002) Model of thalamocortical slow-wave sleep oscillations and transitions to activated states. J Neurosci 22:8691-8704. Medline

Benuskova L, Abraham WC (2007) STDP rule endowed with the BCM sliding threshold accounts for hippocampal heterosynaptic plasticity. J Comput Neurosci 22:129-133. CrossRef Medline

Bi GQ, Poo MM (1998) Synaptic modifications in cultured hippocampal neurons: dependence on spike timing, synaptic strength, and postsynaptic cell type. J Neurosci 18:10464-10472. Medline

Birtoli B, Ulrich D (2004) Firing mode-dependent synaptic plasticity in rat neocortical pyramidal neurons. J Neurosci 24:4935-4940. CrossRef Medline

Bliss TV, Collingridge GL (1993) A synaptic model of memory: long-term potentiation in the hippocampus. Nature 361:31-39. CrossRef Medline

Chen JY, Chauvette S, Skorheim S, Timofeev I, Bazhenov M (2012) Interneuron-mediated inhibition synchronizes neuronal activity during slow oscillation. J Physiol 590:3987-4010. CrossRef Medline

Chen JY, Lonjers P, Lee C, Chistiakova M, Volgushev M, Bazhenov M (2013) Heterosynaptic plasticity prevents runaway synaptic dynamics. J Neurosci 33:15915-15929. CrossRef Medline

Chistiakova M, Volgushev M (2009) Heterosynaptic plasticity in the neocortex. Exp Brain Res 199:377-390. CrossRef Medline

Chistiakova M, Bannon NM, Bazhenov M, Volgushev M (2014) Heterosynaptic plasticity: multiple mechanisms and multiple roles. Neuroscientist 20:483-498. CrossRef Medline

Chistiakova M, Bannon NM, Chen JY, Bazhenov M, Volgushev M (2015) Homeostatic role of heterosynaptic plasticity: models and experiments. Front Comput Neurosci 9:89. CrossRef Medline

Clem RL, Celikel T, Barth AL (2008) Ongoing in vivo experience triggers synaptic metaplasticity in the neocortex. Science 319:101-104. CrossRef Medline

Dunwiddie T, Lynch G (1978) Long-term potentiation and depression of synaptic responses in the rat hippocampus: localization and frequency dependency. J Physiol 276:353-367. CrossRef Medline

Elliott T, Shadbolt NR (2002) Multiplicative synaptic normalization and a nonlinear Hebb rule underlie a neurotrophic model of competitive synaptic plasticity. Neural Comput 14:1311-1322. CrossRef Medline 
Engert F, Bonhoeffer T (1997) Synapse specificity of long-term potentiation breaks down at short distances. Nature 388:279-284. CrossRef Medline

Feldman DE (2009) Synaptic mechanisms for plasticity in neocortex. Annu Rev Neurosci 32:33-55. CrossRef Medline

Fiete IR, Senn W, Wang CZ, Hahnloser RH (2010) Spike-time-dependent plasticity and heterosynaptic competition organize networks to produce long scale-free sequences of neural activity. Neuron 65:563-576. CrossRef Medline

Fonseca R, Nägerl UV, Morris RG, Bonhoeffer T (2004) Competing for memory: hippocampal LTP under regimes of reduced protein synthesis. Neuron 44:1011-1020. CrossRef Medline

Frey U, Morris RG (1997) Synaptic tagging and long-term potentiation. Nature 385:533-536. CrossRef Medline

Frey U, Morris RG (1998) Synaptic tagging: implications for late maintenance of hippocampal long-term potentiation. Trends Neurosci 21: 181-188. CrossRef Medline

Gütig R, Aharonov R, Rotter S, Sompolinsky H (2003) Learning input correlations through nonlinear temporally asymmetric Hebbian plasticity. J Neurosci 23:3697-3714. Medline

Haas JS, Nowotny T, Abarbanel HD (2006) Spike-timing-dependent plasticity of inhibitory synapses in the entorhinal cortex. J Neurophysiol 96: 3305-3313. CrossRef Medline

Hardingham NR, Hardingham GE, Fox KD, Jack JJ (2007) Presynaptic efficacy directs normalization of synaptic strength in layer $2 / 3$ rat neocortex after paired activity. J Neurophysiol 97:2965-2975. CrossRef Medline

Kempter R, Gerstner W, van Hemmen JL (2001) Intrinsic stabilization of output rates by spike-based Hebbian learning. Neural Comput 13: 2709-2741. CrossRef Medline

Lee CM, Stoelzel C, Chistiakova M, Volgushev M (2012) Heterosynaptic plasticity induced by intracellular tetanization in layer $2 / 3$ pyramidal neurons in rat auditory cortex. J Physiol 590:2253-2271. CrossRef Medline

Lemieux M, Chen JY, Lonjers P, Bazhenov M, Timofeev I (2014) The impact of cortical deafferentation on the neocortical slow oscillation. J Neurosci 34:5689-5703. CrossRef Medline

Lynch GS, Dunwiddie T, Gribkoff V (1977) Heterosynaptic depression: a postsynaptic correlate of long-term potentiation. Nature 266:737-739. CrossRef Medline

Mainen ZF, Sejnowski TJ (1996) Influence of dendritic structure on firing pattern in model neocortical neurons. Nature 382:363-366. CrossRef Medline

Miller KD (1996) Synaptic economics: competition and cooperation in synaptic plasticity. Neuron 17:371-374. CrossRef Medline

Miller KM (1994) The role of constraints of Hebbian learning. Neural Comput 6:100-126. CrossRef

Morrison A, Aertsen A, Diesmann M (2007) Spike-timing-dependent plasticity in balanced random networks. Neural Comput 19:1437-1467. CrossRef Medline

Müller W, Swandulla D (1995) Synaptic feedback excitation has hypothalamic neural networks generate quasirhythmic burst activity. J Neurophysiol 73:855-861. Medline

Nevian T, Sakmann B (2006) Spine $\mathrm{Ca}^{2+}$ signaling in spike-timingdependent plasticity. J Neurosci 26:11001-11013. CrossRef Medline

Nishiyama M, Hong K, Mikoshiba K, Poo MM, Kato K (2000) Calcium stores regulate the polarity and input specificity of synaptic modification. Nature 408:584-588. CrossRef Medline

Oja E (1982) A simplified neuron model as a principal component analyzer. J Math Biol 15:267-273. CrossRef Medline

Royer S, Paré D (2003) Conservation of total synaptic weight through balanced synaptic depression and potentiation. Nature 422:518-522. CrossRef Medline

Rubin J, Lee DD, Sompolinsky H (2001) Equilibrium properties of temporally asymmetric Hebbian plasticity. Phys Rev Lett 86:364-367. CrossRef Medline

Schuman EM, Madison DV (1994) Locally distributed synaptic potentiation in the hippocampus. Science 263:532-536. CrossRef Medline

Sjöström PJ, Turrigiano GG, Nelson SB (2001) Rate, timing, and cooperativity jointly determine cortical synaptic plasticity. Neuron 32:1149-1164. CrossRef Medline

Skorheim S, Lonjers P, Bazhenov M (2014a) A spiking network model of decision making employing rewarded STDP. PLoS One 9:e90821. CrossRef Medline

Skorheim S, Razak K, Bazhenov M (2014b) Network models of frequency modulated sweep detection. PLoS One 9:e115196. CrossRef Medline

Song S, Abbott LF (2001) Cortical development and remapping through spike timing-dependent plasticity. Neuron 32:339-350. CrossRef Medline

Song S, Miller KD, Abbott LF (2000) Competitive Hebbian learning through spike-timing-dependent synaptic plasticity. Nat Neurosci 3: 919-926. CrossRef Medline

Song S, Sjöström PJ, Reigl M, Nelson S, Chklovskii DB (2005) Highly nonrandom features of synaptic connectivity in local cortical circuits. PLoS Biol 3:e68. CrossRef Medline

van Ooyen A (2001) Competition in the development of nerve connections: a review of models. Network 12:R1-R47. CrossRef Medline

van Rossum MC, Bi GQ, Turrigiano GG (2000) Stable Hebbian learning from spike timing-dependent plasticity. J Neurosci 20:8812-8821. Medline

Volgushev M, Voronin LL, Chistiakova M, Singer W (1997) Relations between long-term synaptic modifications and paired-pulse interactions in the rat neocortex. Eur J Neurosci 9:1656-1665. CrossRef Medline

Volgushev M, Balaban P, Chistiakova M, Eysel UT (2000) Retrograde signalling with nitric oxide at neocortical synapses. Eur J Neurosci 12:42554267. CrossRef Medline

von der Malsburg C (1973) Self-organization of orientation sensitive cells in the striate cortex. Kybernetik 14:85-100. CrossRef Medline

Yeung LC, Shouval HZ, Blais BS, Cooper LN (2004) Synaptic homeostasis and input selectivity follow from a calcium-dependent plasticity model. Proc Natl Acad Sci U S A 101:14943-14948. CrossRef Medline

Zenke F, Hennequin G, Gerstner W (2013) Synaptic plasticity in neural networks needs homeostasis with a fast rate detector. PLoS Comput Biol 9:e1003330. CrossRef Medline

Zenke F, Agnes EJ, Gerstner W (2015) Diverse synaptic plasticity mechanisms orchestrated to form and retrieve memories in spiking neural networks. Nat Commun 6:6922. CrossRef Medline

Zhou YD, Acker CD, Netoff TI, Sen K, White JA (2005) Increasing $\mathrm{Ca}^{2+}$ transients by broadening postsynaptic action potentials enhances timingdependent synaptic depression. Proc Natl Acad Sci U S A 102:1912119125. CrossRef Medline 